\title{
Analysis of constraints and their algebra in bimetric theory
}

\author{
S.F. Hassan and Anders Lundkvist \\ Department of Physics $\&$ The Oskar Klein Centre, Stockholm University, \\ AlbaNova University Centre, SE-106 91 Stockholm, Sweden \\ E-mail: fawad@fysik.su.se, anders.lundkvist@fysik.su.se
}

\begin{abstract}
We perform a canonical analysis of the bimetric theory in the metric formulation, computing the constraints and their algebra explicitly. In particular, we compute a secondary constraint, that has been argued to exist earlier, and show that it has the correct form to eliminate the ghost. We also identify a set of four first class constraints that generate the algebra of general covariance. The covariance algebra naturally determines a spacetime metric for the theory. However, in bimetric theory, this metric is not unique but depends on how the first class constraints are identified.
\end{abstract}

Keywords: Classical Theories of Gravity, Cosmology of Theories beyond the SM

ARXIV EPRINT: 1802.07267 


\section{Contents}

1 Introduction $\quad 1$

1.1 Background 2

1.2 Summary of results 4

2 Review of constraints in bimetric gravity $\quad 6$

2.1 The Hamiltonian formulation of bimetric theory 6

$\begin{array}{ll}2.2 & \text { Outline of the analysis }\end{array}$

2.3 Constraints from lapse and shift equations 9

$\begin{array}{ll}2.4 & \text { The existence of the extra secondary constraint } \\ \end{array}$

3 The secondary constraint in bimetric theory 13

$\begin{array}{ll}3.1 & \text { Evaluation of the bimetric secondary constraint } \\ \end{array}$

$\begin{array}{ll}3.2 \text { The } L^{i} \text { independence of } \mathcal{C}_{(2)} & 16\end{array}$

$\begin{array}{ll}3.3 \text { The } \partial_{i} L \text { independence of } \mathcal{C}_{(2)} & 16\end{array}$

4 The algebra of general covariance and the HKT metric $\quad 18$

$\begin{array}{ll}4.1 \text { The algebra of general covariance } & 18\end{array}$

$\begin{array}{lll}4.2 & \text { First class constraints in bimetric theory } & 18\end{array}$

$\begin{array}{ll}4.3 \text { The HKT metrics } & 20\end{array}$

A Determining the Lagrange multipliers $\quad 22$

B Computation of the Poisson brackets $\quad 28$

B.1 Evaluation of $\left\{\tilde{R}^{0}(x), \tilde{R}_{i}(y)\right\} \quad 29$

B.2 Evaluation of $\left\{\tilde{R}^{0}(x), \tilde{R}^{0}(y)\right\} \quad 34$

B.3 Poisson brackets with the constraint $\mathcal{C} \quad 36$

\section{Introduction}

Bimetric theory is a theory of the gravitational metric interacting with another spin-2 field. The study of such a theory can be motivated by comparing general relativity with Maxwell's theory and noting that they are the simplest possible theories of a spin- 2 and a spin- 1 field, respectively. However, while Maxwell's theory works perfectly well at the classical level, and to a large extent even at the quantum level, in reality it is not a standalone theory. Rather, it is embeded in the $\mathrm{SU}(2)_{W} \times \mathrm{U}(1)_{Y}$ electroweak theory. In fact, all fields in the standard model appear in multiplets, which is crucial for the consistency and observational viability of the theory. This feature of the standard model suggests the possibility that general relativity could arise from a setup including more spin-2 fields. However, constructing theories of multiple spin-2 fields is not straightforward, since such theories have redundant 
field components, including ghost modes [1], which must be eliminated by appropriate constraints present in the theory.

In this paper we carry out an analysis of constraints in the ghost free bimetric theory $[2,3]$, which is formulated in terms of two spin-2 fields $g_{\mu \nu}$ and $f_{\mu \nu}$. We compute the constraints explicitly, in particular a secondary constraint that was argued to exist in [4], showing that it has the correct form to eliminate the ghost. We also identify a set of four first class constraints that generate the algebra of general covariance. From the work of Hojman, Kuchař and Teitelboim (HKT) it known that one can read off a metric from this algebra [5]. We show that this metric is not unique, but depends on what combination of constraints are chosen to form a first class set.

Ghost free massive gravity [6-8], which can be obtained from bimetric theory by freezing the dynamics of $f_{\mu \nu}$, has fewer constraints, due to the lack of general covariance. These were computed in $[9,10]$, in [4], and subsequently in [11-18]. In bimetric theory, of the six constraints needed, five were obtained in [2] and the sixth one was argued to exist in [4]. More work on this has been carried out in [19-26]. In this paper we address the computation of the sixth constraint and of the algebra of constraints, as detailed below.

The paper is organized as follows. In the remainder of this section we review the known results on bimetric constraints and then summarize the results of the present paper. Section 2 gives a review of the ghost free bimetric theory and presents an outline of the calculations in our reduced phase space approach. In section 3, the secondary constraint of the bimetric theory is evaluated explicitly and shown to have the required form. Section 4 deals with finding the first class constraints in bimetric theory and identifying the HKT metric from the general covariance algebra. In appendix A, all Lagrange multipliers of the theory are determined at the linear level. Detailed derivations of the Poisson brackets of constraints are relegated to appendix B.

\subsection{Background}

We consider a gravitational metric $g_{\mu \nu}$ with an Einstein-Hilbert action, interacting with a similar spin-2 field represented by a symmetric tensor $f_{\mu \nu}$, via a potential $V\left(g^{-1} f\right)$,

$$
\mathcal{S}=\int \mathrm{d}^{4} x\left(M_{g}^{2} \sqrt{|\operatorname{det} g|} R^{(g)}+M_{f}^{2} \sqrt{|\operatorname{det} f|} R^{(f)}+2 m^{4} \sqrt{|\operatorname{det} g|} V\left(g^{-1} f\right)\right) .
$$

Then the necessary condition for the absence of ghosts [27] determines the form of the potential [6-8] which is specified explicitly in section 2.1. It also dictates the dynamics of $f_{\mu \nu}$ to be given by an Einstein-Hilbert term [2] leading to the above bimetric action. To eliminate the ghost modes, the theory must contain appropriate constraints as described below.

In [3] it was shown that general covariance and the reality of the bimetric equations in section 2.1 restrict the two metrics such that their null cones always have a nonvanishing intersection. An implication is that one can always find coordinate systems in which the two metrics admit simultaneous $3+1$ decompositions in terms of their respective lapses, 
shifts and spatial 3-metrics,

$$
\begin{aligned}
N & \equiv\left(-g^{00}\right)^{-1 / 2}, & N_{i} \equiv g_{0 i}, & \gamma_{i j} \equiv g_{i j} \\
L & \equiv\left(-f^{00}\right)^{-1 / 2}, & L_{i} \equiv f_{0 i}, & \phi_{i j} \equiv f_{i j} .
\end{aligned}
$$

This decomposition, used in $[2,10]$, is convenient for analyzing the dynamical content of the theory.

Because of the Einstein-Hilbert terms, only time derivatives of $\gamma_{i j}$ and $\phi_{i j}$ appear in the action, hence there are 12 potentially propagating degrees of freedom. The 8 components of the lapses and shifts are nondynamical. The propagating degrees of freedom are described by the field components $\gamma_{i j}$ and $\phi_{i j}$ and their canonically conjugate momenta, say, $\pi^{i j}$ and $p^{i j}$, in a 24 dimensional phase space. As in any covariant theory, the general covariance of the action gives rise to 4 constraints that can be used to eliminate 4 of the phase space variables. Another 4 can be eliminated by gauge fixing general coordinate transformations. Of the remaining 8 potentially propagating modes (corresponding to 16 phase space field variables), 7 are physical, corresponding to a massless and a massive spin-2 field, and the remaining one is the Boulware-Deser ghost. Since there is no leftover gauge symmetry to eliminate more field components, two more constraints are needed to eliminate the ghost field and its conjugate momentum. These exist for the bimetric theory (1.1) with the potential specified in section 2.1, but not for generic bimetric actions. Hence, in total, we need 6 constraints including the ones associated with general covariance. We also need further equations that determine the lapses and shifts.

For the theory specified in section 2.1, after some manipulations one set of the shift variables, say, the $N_{i}$, can be eliminated using their equations of motion, leading to a Hamiltonian with $N, L$ and $L^{i}$ as Lagrange multipliers, [2, 10],

$$
\mathcal{H}=-\left(N \mathcal{C}+L \tilde{R}^{0}+L^{i} \tilde{R}_{i}\right)
$$

This yields the first five constraints, $\mathcal{C}=0, \tilde{R}^{0}=0$, and $\tilde{R}_{i}=0$. Since these equations arise from an action principle, they are valid at all times, in particular $\dot{\mathcal{C}} \approx 0$, where the symbol $\approx$ is used for weak equalities, i.e. equalities that hold on the surface of the constraints. When expressed in terms of the Poisson bracket, this gives a new condition,

$$
\mathcal{C}_{(2)} \equiv \frac{\mathrm{d} \mathcal{C}}{\mathrm{d} t}=\{\mathcal{C}, H\} \approx 0
$$

This will be the last constraint needed, provided it has the correct structure. We will see that $\tilde{R}_{i}$ are automatically preserved in time while $\mathcal{C}_{(2)} \approx 0$ ensures the preservation of $\tilde{R}^{0}$. For a generic bimetric theory, the Hamiltonian cannot be expressed in the above form and the constraints $\mathcal{C}$ and $\mathcal{C}_{(2)}$ do not exist.

Let us first review the situation in massive gravity. This is given by the action (1.1) without the Einstein-Hilbert term for $f_{\mu \nu}$ which is then treated as a fixed nondynamical metric without a corresponding equation of motion. In this case two constraints are needed to eliminate the ghost. These exist for the potential specified in section 2.1 in which case 
$\mathcal{C} \approx 0$ is obtained directly from the Hamiltonian. Its preservation in time gives,

$$
\mathcal{C}_{(2)}=\frac{\partial \mathcal{C}}{\partial t}+\{\mathcal{C}, H\} \approx 0
$$

The $\partial \mathcal{C} / \partial t$ term accounts for the explicit time dependence via the background $f_{\mu \nu}$ which is no longer computed by the Poisson bracket. It was shown in [4] that in such massive gravity theories one has $\{\mathcal{C}(x), \mathcal{C}(y)\} \approx 0$. Hence, $\mathcal{C}_{(2)}$ is independent of $N$ and provides the needed secondary constraint. The explicit form of $\mathcal{C}_{(2)}$ valid for time independent $f_{\mu \nu}$ was computed in [4]. Furthermore, it was argued that since these properties also hold in bimetric theory, equation (1.5) is a constraint in that case as well. The massive gravity constraints have also been obtained in [11-13] and further confirmed in [14-17] without a $3+1$ decomposition. They were used for the analysis of classical solutions in [28, 29]. Conversely, $[11,12]$ has shown that requiring the existence of appropriate constraints in a generic massive gravity can restrict the potential to the ghost-free cases. Note that $\mathcal{C}_{(2)}$ in massive gravity depends on the lapse $L$ and shift $L_{i}$ of the nondynamical $f_{\mu \nu}$ [4], which is not a problem since these are prespecified functions.

\subsection{Summary of results}

The results of the present paper can now be summarized in the context of the above background discussion.

Constraints in bimetric theory. It was pointed out in $[19,20]$ that also in the bimetric theory specified in section $2.1, \mathcal{C}_{(2)}$, given by (1.5), depends on the lapse $L$ and its spatial derivative $\partial_{i} L$. It was argued that then $\mathcal{C}_{(2)} \approx 0$ is not a constraint since it would determine $L$ rather than eliminate the momentum conjugate to the ghost field. In this paper, we show that this is not the case. For the bimetric theory in (2.1) we perform a canonical analysis in the metric formulation, computing all constraints and their stability conditions. In particular $\mathcal{C}_{(2)}$ is calculated explicitly and shown to contain $L$ only as an overall factor. All terms involving $L^{i}$ and $\partial_{i} L$ are shown to vanish. Hence $\mathcal{C}_{(2)} \approx 0$ is a valid secondary constraint, ensuring the absence of the Boulware-Deser ghost. It is also shown that the stability condition $\dot{\mathcal{C}}_{(2)} \approx 0$ can be solved for $N$ in terms of $L$. Then, $L$ and $L^{i}$ are determined in the process of gauge fixing general coordinate transformations, allowing us to express all Lagrange multipliers in terms of the dynamical variables. This is explicitly worked out at the linear level in appendix A. The explicit expressions obtained for the constraints are needed for the initial value problem.

The canonical analysis of bimetric theory has also been performed in [22, 23], in chiral and tetrad variables, respectively. They conclude that the theory does have the constraints to eliminate the ghost and propagates the appropriate seven degrees of freedom. However, due to the different formalisms used, it not trivial to directly compare their expression for the secondary constraints with the ones obtained here. 
First class constraints and the HKT metric. GR has a Hamiltonian $\mathcal{H}_{G R}=-N^{\mu} R_{\mu}$, leading to constraints $R_{0} \approx 0, R_{i} \approx 0$, which in turn satisfy the general covariance algebra,

$$
\begin{aligned}
& \left\{R^{0}(x), R^{0}(y)\right\}=-\left[R^{i}(x) \frac{\partial}{\partial x^{i}} \delta^{3}(x-y)-R^{i}(y) \frac{\partial}{\partial y^{i}} \delta^{3}(x-y)\right] \\
& \left\{R^{0}(x), R_{i}(y)\right\}=-R^{0}(y) \frac{\partial}{\partial x^{i}} \delta^{3}(x-y) \\
& \left\{R_{i}(x), R_{j}(y)\right\}=-\left[R_{j}(x) \frac{\partial}{\partial x^{i}} \delta^{3}(x-y)-R_{i}(y) \frac{\partial}{\partial y^{j}} \delta^{3}(x-y)\right] .
\end{aligned}
$$

In fact, this is a feature of any covariant theory [30]. The algebra contains both $R_{i}$ and $R^{i}$, hence it explicitly depends on a 3 -metric that relates the two. In GR this is the spatial metric $g_{i j}$.

In [5], Hojman, Kuchař and Teitelboim conjectured that this observation can be used to identify a 3-metric in theories which have general covariance but where a unique metric is not a priori specified. Obviously, bimetric theory is such an example. The theory admits two independent matter sectors each coupled minimally to one of the metrics [2,31]. Therefore, restricting to a matter sector alone, the covariance algebra leads to either $g_{i j}$ or $f_{i j}$ as the HKT metric. However, the full theory can also contain other combinations of $g$ and $f$ as effective metrics, and a priori it is not obvious if a preferred combination exists and how this is compatible with the matter sectors.

In [22], the constraint algebra was studied in a chiral formulation of the bimetric theory. The metric identified using the HKT-conjecture is a complicated function of the variables used and does not coincide with either $g_{\mu \nu}$ or $f_{\mu \nu}$. Some aspects of the bimetric constraint algebra have been considered in [24, 25], in [19-21], as well as in [23], but without addressing the HKT metric.

Having obtained the constraints, in this paper we also compute the constraint algebra. We identify a set of 4 first class constraints among the six outlined above, and show that they satisfy the standard algebra of general covariance. The first class constraints can be easily identified by imposing the second class constraints and the stability condition $\dot{\mathcal{C}}_{2} \approx 0$, to bring the bimetric Hamiltonian to the form $\mathcal{H}=-L^{\mu} R_{\mu}$. The HKT metric appearing in the covariance algebra is then found to be $f_{i j}$. However, using an alternative set of variables modifies the first class constraints and leads to $g_{i j}$ as the HKT metric. The explanation is that a set of first class constraints can be identified among all the constraints in different ways, each choice leading to a different HKT metric. The possibility of other choices, beyond the two mentioned above, is also discussed. It is possible that the metric identified in [22] corresponds to one such choice. All this is consistent with matter couplings, since their structure is similar to the gravitational sector.

In this paper the constraints have mainly been used to argue for the absence of ghosts and the correct counting of degrees of freedom. In addition, they are also essential for determining valid initial data that will be evolved by the remaining dynamical equations. However, for bimetric theory to be fully consistent, it is also necessary that the dynamical equations produce causal evolution. Some aspects of this problem have been investigated in $[3,32-37]$. 


\section{Review of constraints in bimetric gravity}

In this section we review the derivation of the constraints in bimetric theory following $[2,4]$.

\subsection{The Hamiltonian formulation of bimetric theory}

The most general ghost free bimetric action for spin-2 fields $g_{\mu \nu}$ and $f_{\mu \nu}$ is [2],

$$
\mathcal{S}=\int \mathrm{d}^{4} x\left(M_{g}^{2} \sqrt{|\operatorname{det} g|} R^{(g)}+M_{f}^{2} \sqrt{|\operatorname{det} f|} R^{(f)}+2 m^{4} \sqrt{|\operatorname{det} g|} \sum_{n=0}^{4} \beta_{n} e_{n}\left(\sqrt{g^{-1} f}\right)\right),
$$

where $R^{(g)}$ and $R^{(f)}$ are the respective Ricci scalars, $M_{g}$ and $M_{f}$ are the corresponding Planck masses, and $\beta_{n}$ are five free parameters. The interactions between $g_{\mu \nu}$ and $f_{\mu \nu}$ involve the square root matrix $S=\sqrt{g^{-1} f}$, which is a specific root of the matrix $g^{-1} f$ to be specified below. The $e_{k}(S)$ are elementary symmetric polynomials of the eigenvalues of $S$ given by $($ with $\operatorname{Tr}(S)=[S])$,

$$
\begin{aligned}
& e_{0}(S)=1, \quad e_{1}(S)=[S], \quad e_{2}(S)=\frac{1}{2}\left([S]^{2}-\left[S^{2}\right]\right), \quad e_{3}(S)=\frac{1}{6}\left([S]^{3}-3[S]\left[S^{2}\right]+2\left[S^{3}\right]\right), \\
& e_{4}(S)=\frac{1}{24}\left([S]^{4}-6[S]^{2}\left[S^{2}\right]+3\left[S^{2}\right]^{2}+8[S]\left[S^{3}\right]-6\left[S^{4}\right]\right) .
\end{aligned}
$$

For a $4 \times 4$ matrix, $e_{4}(S) \equiv \operatorname{det} S$ and $e_{k}(S) \equiv 0$ for $k>4$. Note that $\operatorname{det}(1+S)=\sum_{n=0}^{4} e_{n}(S)$, so the potential $\sum_{n=0}^{4} \beta_{n} e_{n}(S)$ is a deformation of $\operatorname{det}(1+S)$. Each term has the property,

$$
\sqrt{|\operatorname{det} g|} e_{n}\left(\sqrt{g^{-1} f}\right)=\sqrt{|\operatorname{det} f|} e_{4-n}\left(\sqrt{f^{-1} g}\right),
$$

hence, the action (2.1) retains its form under the interchange of $g_{\mu \nu}$ and $f_{\mu \nu}[2]$. The theory can be easily generalized to any dimension $d$.

By definition, the matrix $S=\sqrt{g^{-1} f}$ must satisfy $[7,8]$,

$$
\left(\sqrt{g^{-1} f}\right)_{\lambda}^{\mu}\left(\sqrt{g^{-1} f}\right)_{\nu}^{\lambda}=g^{\mu \lambda} f_{\lambda \nu}
$$

This does not specify the square root uniquely and may even admit nonreal solutions. Obviously, as long as $S$ is not uniquely specified, the action (2.1) remains ill defined. This problem has a natural resolution as follows (see [3] for details). In order for the action to be invariant under coordinate transformations, $S^{\mu}{ }_{\nu}$ must transform as a $(1,1)$ tensor and only the principal root has this property. ${ }^{1}$ Furthermore, to obtain real equations of motion, $g$ and $f$ must be restricted such that a real principal root always exists. This restriction turns out to imply that the null cones of the metrics $g$ and $f$ have a nonvanishing intersection, so that they admit common spacelike and timelike directions [3]. Thus, the requirements of reality and general covariance specify the theory uniquely by restricting $S$ to the real principal root of $g^{-1} f$. The same requirements also ensure that all allowed $g$ and $f$ admit simultaneous proper $3+1$ decompositions.

\footnotetext{
${ }^{1}$ All non-principal roots cease to transform as $(1,1)$ tensors whenever eigenvalues of $g^{-1} f$ belonging to distinct Jordan blocks happen to coincide.
} 
Therefore, without loss of generality, we can use the $3+1$ metric decompositions (1.2) and (1.3) in the bimetric action. As in GR, the action will only contain time derivatives of the 3 -metrics $\gamma_{i j}$ and $\phi_{i j}$. Denoting the corresponding canonical momenta by $\pi^{i j}$ and $p^{i j}$, the Lagrangian can be readily expressed in terms of the phase space variables, as in [38],

$$
\begin{aligned}
\mathcal{L}= & \left(\pi^{i j} \dot{\gamma}_{i j}+N R^{0(g)}+N^{i} R_{i}^{(g)}\right)+\left(p^{i j} \dot{\phi}_{i j}+L R^{0(f)}+L^{i} R_{i}^{(f)}\right) \\
& +2 m^{4} N \sqrt{\operatorname{det} \gamma} \sum_{n=0}^{3} \beta_{n} e_{n}\left(\sqrt{g^{-1} f}\right) .
\end{aligned}
$$

The first line simply contains the $3+1$ decomposition of the Einstein-Hilbert terms for $g$ and $f$. Here, $N^{i}=\gamma^{i j} N_{j}$ and $L^{i}=\phi^{i j} L_{j}$, where $\gamma^{i j}$ and $\phi^{i j}$ are the inverses of $\gamma_{i j}$ and $\phi_{i j}$, respectively. As in general relativity, $R^{0(g)}, R_{i}^{(g)}, R^{0(f)}$ and $R_{i}^{(f)}$ are given by,

$$
\begin{aligned}
& R^{0(g)}=M_{g}^{2} \sqrt{\operatorname{det} \gamma}^{3} R^{(g)}+\frac{1}{M_{g}^{2} \sqrt{\operatorname{det} \gamma}}\left(\frac{1}{2} \pi^{2}-\pi^{i j} \pi_{i j}\right), \quad R_{i}^{(g)}=2 \sqrt{\operatorname{det} \gamma} \gamma_{i j} \nabla_{k}\left(\frac{\pi^{j k}}{\sqrt{\operatorname{det} \gamma}}\right), \\
& R^{0(f)}=M_{f}^{2} \sqrt{\operatorname{det} \phi}^{3} R^{(f)}+\frac{1}{M_{f}^{2} \sqrt{\operatorname{det} \phi}}\left(\frac{1}{2} p^{2}-p^{i j} p_{i j}\right), \quad R_{i}^{(f)}=2 \sqrt{\operatorname{det} \phi} \phi_{i j} \bar{\nabla}_{k}\left(\frac{p^{j k}}{\sqrt{\operatorname{det} \phi}}\right),
\end{aligned}
$$

where $\nabla_{i}$ and $\bar{\nabla}_{i}$ are the covariant derivatives compatible with $\gamma_{i j}$ and $\phi_{i j}$, respectively.

In (2.5), the lapses and shifts, $N, L, N^{i}$ and $L^{i}$, appear without time derivatives. The dynamical variables are $\gamma_{i j}, \phi_{i j}, \pi^{i j}$ and $p^{i j}$ with a total of 24 (phase space) components, corresponding to 12 potentially propagating degrees of freedom, including potential ghost modes. $^{2}$ The theory has enough symmetries and constraints to eliminate the ghost and reduce the number of propagating modes to seven, corresponding to a massless and a massive spin-2 field. However, since the potential term in (2.5) is nonlinear in the lapses and shifts, some manipulations are needed to make the constraints manifest. This is outlined in subsection 2.3, where the constraint that eliminates the ghost field is obtained. A second constraint that eliminates the canonical momentum of the ghost field is computed in section 3 .

\subsection{Outline of the analysis}

To obtain the constraint equations, we work in a reduced phase space. Before getting into the details, it is worthwhile to briefly outline our procedure and show, in particular, that it is equivalent to the more elaborate formalism of [11, 12, 19-21] involving an enlarged phase space. In the next subsection we will see that the bimetric Lagrangian has the general form $L\left(q_{m}, \dot{q}_{m}, Q_{r}, n_{i}\right)$, depending on a set of dynamical variables $q_{m}(t)$ and two sets of nondynamical variables $Q_{r}(t)$ and $n_{i}(t)$. Here, for simplicity we ignore the space dependence of fields and gauge invariances. The nonvanishing canonical momenta are

\footnotetext{
${ }^{2}$ In this usage, a degree of freedom consists of two phase space variables, i.e., the field and its conjugate momentum.
} 
$p^{m}=\partial L / \partial \dot{q}_{m}$ conjugate to $q_{m}$. The Lagrangian equations of motion are,

$$
\dot{p}^{m}=\frac{\partial L}{\partial q_{m}}, \quad \frac{\partial L}{\partial Q_{m}}=0, \quad \frac{\partial L}{\partial n_{i}}=0 .
$$

These contain all the information about dynamics and constraints. To completely disentangle the two, we rewrite the action in terms of phase space variables using the Hamiltonian $H=p^{m} \dot{q}_{m}-L$,

$$
S=\int d t\left[p^{m} \dot{q}_{m}-H(p, q, Q, n)\right]
$$

Then, on varying with respect to $q_{m}, p^{m}, Q_{r}$, and $n_{i}$, one obtains the equivalent set of equations,

$$
\dot{p}^{m}=-\frac{\partial H}{\partial q_{m}}, \quad \dot{q}_{m}=\frac{\partial H}{\partial p^{m}}, \quad \frac{\partial H}{\partial Q_{m}}=0, \quad \frac{\partial H}{\partial n_{i}}=0 .
$$

It turns out that the $n_{i}$ equations determine $n_{i}=n_{i}(q, p)$, independent of $Q_{r}$, which can be used to eliminate the $n_{i}$ from the action. Although now the action develops an extra dependence on $p^{m}$ and $q_{m}$ through the $n_{i}$, in practice, variations of $H$ can be computed at fixed $n_{i}$, since,

$$
\delta H=\left.\delta\right|_{n_{i}} H+\left.\frac{\partial H}{\partial n_{i}}\right|_{q_{m}, p^{m}, Q_{r}} \delta n_{i},
$$

and the last term vanishes for the solutions of the $n_{i}$ equations. Hence, effectively, $H=H(q, p, Q)$. In bimetric theory, the $Q_{r}$ appear as Lagrange multipliers and the Hamiltonian has the form $H=\mathcal{H}_{r}(p, q) Q_{r}$. It follows that $\partial \mathcal{H}_{r} / \partial n_{i}=0$, either being proportional to the $n_{i}$ equations of motion or because some $\mathcal{H}_{r}$ did not contain $n_{i}$ to begin with. In terms of the Poisson brackets defined with respect to the conjugate pairs $\left(q_{m}, p^{m}\right)$, the above equations can be recast as,

$$
\dot{p}^{m}=\left\{p^{m}, H\right\}, \quad \dot{q}^{m}=\left\{q^{m}, H\right\}, \quad \mathcal{H}_{r}(p, q)=0 .
$$

The constraints on $\mathcal{H}_{r}$ can be solved to eliminate an equal number of dynamical variables.

In bimetric theory the above equations hide one more constraint that can be extracted as follows. Since the constraints are obtained from the action principle, they hold at all times, in particular, $d \mathcal{H}_{r} / d t=0$. One of the constraints, say $\mathcal{H}_{1} \equiv C$ is special. Since $C$ varies only with $p^{m}$ and $q_{m}$, we get $d C / d t=\{C, H\}$, using (2.10). Hence, $\dot{C}=0$ combined with (2.10) implies,

$$
C_{2} \equiv\{C, H\}=0
$$

This turns out to be independent of the $Q_{r}$ and thus is a new constraint. One can also compute $d C_{2} / d t=\left\{C_{2}, H\right\}=0$, but this equation contains some of the $Q_{r}$ and is not a constraint on the $q_{m}$ and $p^{m}$. The remaining $\mathcal{H}_{r}$ do not lead to new constraints in this way. Note that, although expressed in terms of Poisson brackets, the above equations are derived from the action principle. 
Let's now compare the above analysis to the formalism of constrained Hamiltonian dynamics applied to the same problem [11, 12, 19-21]. This is based on an enlarged phase space spanned by variables $q_{m}, Q_{r}$ and their conjugate momenta $p^{m}, P^{r}$, where the primary constraints $P^{r}=0$ are enforced through Lagrange multipliers $u_{r}$. The relevant Hamiltonian is,

$$
H^{\prime}=H(p, q, Q)+P^{r} u_{r}
$$

We can easily extend this to also include the nondynamical variables $n_{i}$ and their conjugate momenta, say $m^{i}=0$. Equations of motion are now given in terms of the Poisson brackets with $H^{\prime}$ on the enlarged phase space. One can easily check that $\dot{p}^{m}=\left\{p^{m}, H^{\prime}\right\}^{\prime}$ and $\dot{q}^{m}=\left\{q^{m}, H^{\prime}\right\}^{\prime}$ reduce to the corresponding equations in the reduced formalism given above. The preservation of the primary constraints in time then gives $\dot{P}^{r}=\left\{P^{r}, H^{\prime}\right\}^{\prime}=0$ which reproduce the constraints $\mathcal{H}_{i}=0$. If $n_{i}$ are included, their equations of motion will also arise in this way. The new equations are $\dot{Q}_{r}=\left\{Q_{r}, H^{\prime}\right\}^{\prime}=u_{r}$ which reflect the fake dynamics of the $Q_{r}$. Finally, the preservation of $C=\mathcal{H}_{1}$ in time leads to the same constraint $C_{2} \equiv \dot{C}=\left\{C, H^{\prime}\right\}^{\prime}=0$ as above, and the $\dot{C}_{2}=0$ equation is unchanged as can be checked by using the properties of the other constraints. Hence working in the reduced phase space is equivalent to the framework of the constrained Hamiltonian dynamics.

\subsection{Constraints from lapse and shift equations}

In order to deal with the square root matrix in the bimetric action and show that the equations for the eight lapse and shift variables encode five constraints, it is convenient to introduce the shift like variables $n^{i}$ through $[7,9,10],{ }^{3}$

$$
N^{i}-L^{i}=L n^{i}+N D^{i}{ }_{j} n^{j} .
$$

The $3 \times 3$ matrix $D$ can be obtained explicitly as a solution to the equation [10],

$$
\sqrt{x} D=\sqrt{\left(\gamma^{-1}-D n n^{T} D^{T}\right) \phi}
$$

Such a solution always exists [39], but is not needed here. The quantity $x$ stands for,

$$
x \equiv 1-n^{i} \phi_{i j} n^{j} .
$$

From equation (2.16), it follows that $D$ has the property,

$$
\phi_{i k} D_{j}^{k}=\phi_{j k} D^{k}
$$

Now, on eliminating $N^{i}$ in favor of $n^{i}$, the action corresponding to (2.5) becomes, [2]

$$
\mathcal{S}=\int \mathrm{d}^{4} x\left(\pi^{i j} \partial_{t} \gamma_{i j}+p^{i j} \partial_{t} \phi_{i j}+L^{i} \tilde{R}_{i}+L \tilde{R}^{0}+N \mathcal{C}\right)
$$

\footnotetext{
${ }^{3}$ In terms of the original variables, the bimetric potential has a highly nonlinear dependence on the shifts $N$ and $L$ through the square root matrix, which would complicate the identification of the constraints. The parameterization in terms of $n^{i}$ is designed to simplify the dependence on the shift variables [9]. Due to the interchange symmetry (2.3), the subsequent analysis also holds with the roles of $g$ and $f$ interchanged.
} 
where $N, L$ and $L^{i}$ appear as five Lagrange multipliers and we have defined,

$$
\begin{aligned}
\tilde{R}^{0} & =R^{0(f)}+n^{i} R_{i}^{(g)}+2 m^{4} \sqrt{\operatorname{det} \gamma} U^{\prime}, \\
\tilde{R}_{i} & =R_{i}^{(g)}+R_{i}^{(f)}, \\
\mathcal{C} & =R^{0(g)}+R_{i}^{(g)} D^{i}{ }_{j} n^{j}+2 m^{4} \sqrt{\operatorname{det} \gamma} V,
\end{aligned}
$$

with,

$$
\sqrt{\operatorname{det} \gamma} U^{\prime}=\sqrt{\operatorname{det} \gamma} U+\beta_{4} \sqrt{\operatorname{det} \phi}
$$

$U$ and $V$ contain the parameters $\beta_{n}$, and are given by,

$$
\begin{aligned}
U \equiv & \beta_{1} \sqrt{x}+\beta_{2}\left[x e_{1}(D)+n^{i} \phi_{i j} D^{j}{ }_{k} n^{k}\right] \\
& +\beta_{3}\left[\sqrt{x}\left(e_{1}(D) n^{i} \phi_{i j} D^{j}{ }_{k} n^{k}-D^{i}{ }_{k} n^{k} \phi_{i j} D^{j}{ }_{l} n^{l}\right)+x^{3 / 2} e_{2}(D)\right], \\
V \equiv & \beta_{0}+\beta_{1} \sqrt{x} e_{1}(D)+\beta_{2} x e_{2}(D)+\beta_{3} x^{3 / 2} e_{3}(D) .
\end{aligned}
$$

The $e_{n}(D)$ are defined similar to $(2.2)$, but now $e_{3}(D)=\operatorname{det} D$, since $D$ is a $3 \times 3$ matrix.

In the action (2.19), the equations of motion for $N, L$ and $L^{i}$ are $\mathcal{C}=0, \tilde{R}^{0}=0$ and $\tilde{R}_{i}=0$. However, $\mathcal{C}$ and $\tilde{R}^{0}$ depend on the redefined shifts $n^{i}$. Therefore, in order for them to impose constraints on the dynamical variables, it is necessary that the equations for $n^{i}$ are independent of the Lagrange multipliers. Only then will $\mathcal{C}, \tilde{R}^{0}$ and $\tilde{R}_{i}$ impose five constraints on the dynamical variables. Indeed, the $n^{k}$ equations of motion are,

$$
\frac{\partial \mathcal{L}}{\partial n^{k}}=L \frac{\partial \tilde{R}^{0}}{\partial n^{k}}+N \frac{\partial \mathcal{C}}{\partial n^{k}}=0
$$

But, from equation (2.16), it follows that [10],

$$
\frac{\partial \tilde{R}^{0}}{\partial n^{k}}=\mathcal{C}_{k}, \quad \frac{\partial \mathcal{C}}{\partial n^{k}}=\mathcal{C}_{i} \frac{\partial\left(D^{i}{ }_{j} n^{j}\right)}{\partial n^{k}},
$$

where

$$
\begin{aligned}
\mathcal{C}_{i}=R_{i}^{(g)}-2 & m^{4} \sqrt{\operatorname{det} \gamma} \frac{n^{l} \phi_{l j}}{\sqrt{x}}\left[\beta_{1} \delta^{j}{ }_{i}+\beta_{2} \sqrt{x}\left(\delta^{j}{ }_{i} D^{m}{ }_{m}-D^{j}{ }_{i}\right)\right. \\
& \left.+\beta_{3} x\left(\frac{1}{2} \delta^{j}{ }_{i}\left(D^{m}{ }_{m} D^{n}{ }_{n}-D^{m}{ }_{n} D^{n}{ }_{m}\right)+D^{j}{ }_{m} D^{m}{ }_{i}-D^{j}{ }_{i} D^{m}{ }_{m}\right)\right] .
\end{aligned}
$$

Inserting (2.27) into (2.26) yields the equation,

$$
\mathcal{C}_{i}\left[L \delta^{i}{ }_{k}+N \frac{\partial\left(D^{i}{ }_{j}^{j}\right)}{\partial n^{k}}\right]=0
$$

The expression inside the square brackets is the Jacobian matrix $\partial N^{i} / \partial n^{k}$ of (2.15), and is invertible. Hence, the $n^{i}$ equations of motion imply, ${ }^{4}$

$$
\mathcal{C}_{i}=0 .
$$

\footnotetext{
${ }^{4}$ Since $\partial \mathcal{L} / \partial n^{k}=\left(\partial \mathcal{L} / \partial N^{i}\right)\left(\partial N^{i} / \partial n^{k}\right),(2.30)$ are the equations of motion for $N^{i}$ expressed in terms of $n^{i}$.
} 
These are independent of $N, L$ and $L^{i}$ and can in principle be solved for the $n^{i}$. In the $\beta_{1^{-}}$ model (i.e. when $\beta_{2}=\beta_{3}=0$ ) it is easy to obtain the explicit solution, while perturbative solutions can be constructed for general $\beta_{n}$ [10]. When these solutions are inserted in the equations of motion for $N, L$ and $L^{i}$, one obtains five constraints on $\gamma_{i j}, \phi_{i j}, \pi^{i j}$ and $p^{i j}$,

$$
\mathcal{C}=0, \quad \tilde{R}^{0}=0, \quad \tilde{R}_{i}=0 .
$$

$\tilde{R}^{0}$ and $\tilde{R}_{i}$ can be associated with general covariance while $\mathcal{C}=0$ eliminates the BD ghost field. A new constraint is needed to remove the momentum conjugate to the ghost and is reviewed below.

\subsection{The existence of the extra secondary constraint}

The five constraints (2.31) obtained from an action principle are valid at all times, in particular, $\dot{\mathcal{C}} \equiv d \mathcal{C} / d t=0$ holds. Contrast this with the Hamiltonian formulation where such conditions must be imposed additionally, leading to new constraints (see for example, [40]). However, even here recasting in Hamiltonian form, $\dot{\mathcal{C}}=\{\mathcal{C}, H\}$, is useful as it requires eliminating $\dot{\gamma}_{i j}$, etc., using the dynamical equations. Then $\dot{\mathcal{C}}=0$ extracts a new secondary constraint from the dynamical equations. Specifically, if $\mathcal{C}_{(2)} \equiv\{\mathcal{C}, H\} \approx 0$ does not involve Lagrange multipliers, it will be a new constraint on the dynamical variables (otherwise it could be solved for a Lagrange multiplier). ${ }^{5}$

The explicit expression for $\mathcal{C}_{(2)}$ in massive gravity with a fixed $f_{\mu \nu}$ was obtained in [4]. It is independent of $N$, but contains $L$ and $L^{i}$. This is not an issue since in massive gravity these are prespecified functions and $\mathcal{C}_{(2)} \approx 0$ remains a constraint on the dynamical variables. However, in bimetric theory, where $f_{\mu \nu}$ is not prespecified, such a dependence is problematic. Indeed, in $[19,20]$ it was argued that, in bimetric theory, $\mathcal{C}_{(2)}$ depends on $L$ and $\partial_{i} L$ (as one may expect from (2.34)) and hence is no longer a constraint on dynamical variables. Note that, in principle, $\mathcal{C}_{(2)}$ could still turn out to be a constraint since $L$ and $L_{i}$ can be determined in terms of dynamical variables through gauge fixing general covariance.

In the remainder of this section, we review the argument in [4] that a secondary constraint exists in both theories. Then, in section 3 we will go beyond [4] to explicitly evaluate $\mathcal{C}_{(2)}$ in bimetric theory and show that its vanishing does not depend on $L$ and $L_{i}$, hence it is a genuine constraint. We also show that no further constraints arise since $\dot{\mathcal{C}}_{(2)} \approx 0$ determines the Lagrange multiplier $N . L$ and $L_{i}$ are determined through gauge fixing, similarly to what is done in GR. At the linear level, this is explicitly worked out in appendix A.

In bimetric theory $\mathcal{C}$ depends on the dynamical variables, hence using Poisson brackets one has,

$$
\mathcal{C}_{(2)}(x)=\frac{d}{d t} \mathcal{C}(x)=\{\mathcal{C}(x), H\} \approx 0 .
$$

The Hamiltonian can be read off from the action in (2.19) as,

$$
H=-\int \mathrm{d}^{3} y\left(N(y) \mathcal{C}(y)+L(y) \tilde{R}^{0}(y)+L^{i}(y) \tilde{R}_{i}(y)\right) .
$$

\footnotetext{
${ }^{5}$ In the terminology of [40], the vanishing of the momenta conjugate to the lapses and shifts are the primary constraints, and the validity of these at all times leads to (2.31) as secondary constraints. The validity of $\mathcal{C}=0$ at all times gives the new secondary constraint $\mathcal{C}_{(2)}=0$.
} 
Then it follows that,

$$
\mathcal{C}_{(2)}(x) \approx-\int \mathrm{d}^{3} y\left(N(y)\{\mathcal{C}(x), \mathcal{C}(y)\}+L^{i}(y)\left\{\mathcal{C}(x), \tilde{R}_{i}(y)\right\}+L(y)\left\{\mathcal{C}(x), \tilde{R}^{0}(y)\right\}\right) \approx 0,
$$

where the Poisson bracket is defined by

$$
\begin{aligned}
\{A, B\}= & \int \mathrm{d}^{3} z\left(\frac{\delta A}{\delta \gamma_{m n}(z)} \frac{\delta B}{\delta \pi^{m n}(z)}-\frac{\delta A}{\delta \pi^{m n}(z)} \frac{\delta B}{\delta \gamma_{m n}(z)}\right) \\
& +\int \mathrm{d}^{3} z\left(\frac{\delta A}{\delta \phi_{m n}(z)} \frac{\delta B}{\delta p^{m n}(z)}-\frac{\delta A}{\delta p^{m n}(z)} \frac{\delta B}{\delta \phi_{m n}(z)}\right) .
\end{aligned}
$$

Note that if $\{\mathcal{C}(x), \mathcal{C}(y)\} \not \approx 0$, then $(2.34)$ could determine $N$, rather than impose a constraint on the dynamical variables, as suggested in [19]. Hence, for $\mathcal{C}_{(2)}$ to be a constraint, it is necessary that $\{\mathcal{C}(x), \mathcal{C}(y)\} \approx 0$, while in bimetric theory (2.34) must also be independent of $L$ and $L_{i}$. This bracket is evaluated using (2.35), but since $\mathcal{C}$ does not depend on $p^{i j}$ (neither explicitly nor through the $n^{i}$ ), the second line vanishes and the bracket is given by the first line alone. The variations also include the dependence of $\mathcal{C}$ on $\gamma_{i j}$ and $\pi^{i j}$ through $n^{i}$. However, since $\partial \mathcal{C} / \partial n^{k}=0$ by (2.27) and (2.30), the $n^{i}$ dependence can be ignored. Then, using (2.22), one gets [4],

$$
\begin{aligned}
\{\mathcal{C}(x), \mathcal{C}(y)\}= & \left\{R^{0(g)}(x), R^{0(g)}(y)\right\}+\left\{R_{i}^{(g)}(x), R_{j}^{(g)}(y)\right\} D^{i}{ }_{k} n^{k}(x) D^{j}{ }_{l} n^{l}(y) \\
& +\left\{R^{0(g)}(x), R_{i}^{(g)}(y)\right\} D^{i}{ }_{k} n^{k}(y)-\left\{R^{0(g)}(y), R_{i}^{(g)}(x)\right\} D^{i}{ }_{k} n^{k}(x) \\
& +S^{m n}(x) \frac{\delta R_{i}^{(g)}(y)}{\delta \pi^{m n}(x)} D^{i}{ }_{k} n^{k}(y)-S^{m n}(y) \frac{\delta R_{i}^{(g)}(x)}{\delta \pi^{m n}(y)} D^{i}{ }_{k} n^{k}(x)
\end{aligned}
$$

where

$$
S^{m n}=R_{j}^{(g)} \frac{\partial\left(D^{j}{ }_{k} n^{k}\right)}{\partial \gamma_{m n}}+2 m^{4} \frac{\partial(\sqrt{\operatorname{det} \gamma} V)}{\partial \gamma_{m n}} .
$$

From equation (2.16), the following relations can be derived,

$$
\begin{aligned}
\frac{\partial}{\partial \gamma_{m n}} \operatorname{Tr}(\sqrt{x} D) & =-\frac{1}{\sqrt{x}}\left(n^{i} \phi_{i j} \frac{\partial\left(D^{j}{ }_{k} n^{k}\right)}{\partial \gamma_{m n}}-\frac{1}{2} \phi_{i j}\left(D^{-1}\right)_{k}^{j} \frac{\partial \gamma^{k i}}{\partial \gamma_{m n}}\right), \\
\frac{\partial}{\partial \gamma_{m n}}\left(\operatorname{Tr}(\sqrt{x} D)^{2}\right) & =-2\left(n^{i} \phi_{i j} D^{j}{ }_{k} \frac{\partial\left(D^{k}{ }_{l} n^{l}\right)}{\partial \gamma_{m n}}-\frac{1}{2} \phi_{i j} \frac{\partial \gamma^{j i}}{\partial \gamma_{m n}}\right), \\
\frac{\partial}{\partial \gamma_{m n}}\left(\operatorname{Tr}(\sqrt{x} D)^{3}\right) & =-3 \sqrt{x}\left(n^{i} \phi_{i j} D^{j}{ }_{k} D^{k}{ }_{l} \frac{\partial\left(D^{l}{ }_{r} n^{r}\right)}{\partial \gamma_{m n}}-\frac{1}{2} \phi_{i j} D^{j}{ }_{k} \frac{\partial \gamma^{k i}}{\partial \gamma_{m n}}\right) .
\end{aligned}
$$

Using these, eliminating $R_{j}^{(g)}$ though (2.28), and imposing equation (2.30), $S^{m n}$ can be written as

$$
S^{m n}=m^{4} \sqrt{\operatorname{det} \gamma}\left(V \gamma^{m n}-\bar{V}^{m n}\right)
$$

where,

$$
\begin{aligned}
\bar{V}^{m n}=\gamma^{m i} & {\left[\beta_{1} \frac{1}{\sqrt{x}} \phi_{i k}\left(D^{-1}\right)_{j}^{k}+\beta_{2}\left(\phi_{i k}\left(D^{-1}\right)_{j}^{k} D_{l}^{l}-\phi_{i j}\right)\right.} \\
& \left.+\beta_{3} \sqrt{x}\left(\phi_{i k} D^{k}{ }_{j}-\phi_{i j} D^{k}{ }_{k}+\frac{1}{2} \phi_{i k}\left(D^{-1}\right)_{j}^{k}\left(D_{l}^{l} D^{h}{ }_{h}-D^{l}{ }_{h} D^{h}{ }_{l}\right)\right)\right] \gamma^{j n} .
\end{aligned}
$$


Finally, using this, as well as the Poisson brackets (1.7), one gets,

$$
\{\mathcal{C}(x), \mathcal{C}(y)\}=-\left[\mathcal{C}(x) D^{i}{ }_{j} n^{j}(x) \frac{\partial}{\partial x^{i}} \delta^{3}(x-y)-\mathcal{C}(y) D^{i}{ }_{j} n^{j}(y) \frac{\partial}{\partial y^{i}} \delta^{3}(x-y)\right] .
$$

Hence, $\{\mathcal{C}(x), \mathcal{C}(y)\} \approx 0$ which shows the existence of the secondary constraint $\mathcal{C}_{(2)} \approx 0$ in massive gravity, and potentially in bimetric theory [4]. $\mathcal{C}_{(2)}$ is given by the remaining terms in (2.34).

\section{The secondary constraint in bimetric theory}

Here we compute the secondary constraint $\mathcal{C}_{(2)}$ in bimetric theory and show that its vanishing is independent of the lapse and shift functions. We also show that it reduces to the known expression for massive gravity.

\subsection{Evaluation of the bimetric secondary constraint}

Using (2.41), the expression (2.34) for $\mathcal{C}_{(2)}$ on the constraint surface becomes,

$$
\begin{aligned}
\mathcal{C}_{(2)}(z) & \approx-\int \mathrm{d}^{3} y\left\{\mathcal{C}(z), L^{i}(y) \tilde{R}_{i}(y)+L(y) \tilde{R}^{0}(y)\right\} \\
& =-\int \mathrm{d}^{3} y\left(\left\{\mathcal{C}(z), L^{i}(y) \tilde{R}_{i}(y)+L(y) \tilde{R}^{0}(y)\right\}_{g}+\left\{\mathcal{C}(z), L^{i}(y) \tilde{R}_{i}(y)+L(y) \tilde{R}^{0}(y)\right\}_{f}\right)
\end{aligned}
$$

In the second line, the brackets \{\}$_{g}$ are evaluated with respect to $\left(\gamma_{i j}, \pi^{i j}\right)$, and \{\}$_{f}$ are evaluated with respect to $\left(\phi_{i j}, p^{i j}\right)$. Note that, as before, the dependence of $\mathcal{C}$ and $\tilde{R}^{0}$ on $\gamma_{i j}, \pi^{i j}$ and $\phi_{i j}$ through $n^{i}$ can be ignored, due to (2.27). The brackets are therefore evaluated at fixed $n^{i}$. By inspecting (2.20) and (2.21), we observe that the \{\}$_{g}$ bracket coincides with a Poisson bracket computed in [4] for massive gravity, since the new terms in $L^{i} \tilde{R}_{i}+L \tilde{R}^{0}$ that contain $R^{0(f)}$ and $R_{i}^{(f)}$ do not contribute to the bracket. This contribution is given by,

$$
\begin{aligned}
\int \mathrm{d}^{3} y & \left\{\mathcal{C}(z), L^{i}(y) \tilde{R}_{i}(y)+L(y) \tilde{R}^{0}(y)\right\}_{g}=-\left(\frac{m^{4}}{M_{g}^{2}} L\left(\gamma_{m n} \pi^{k}{ }_{k}-2 \pi_{m n}\right) U^{m n}\right. \\
& +2 m^{4} \sqrt{\operatorname{det} \gamma} \gamma_{n i} D^{i}{ }_{k} n^{k} \nabla_{m}\left(L U^{m n}\right)+\left(R_{j}^{(g)} D^{i}{ }_{k} n^{k}-2 m^{4} \sqrt{\operatorname{det} \gamma} \gamma_{j k} \bar{V}^{k i}\right) \nabla_{i}\left(L n^{j}+L^{j}\right) \\
& \left.+\sqrt{\operatorname{det} \gamma}\left[\nabla_{i}\left(\frac{R^{0(g)}}{\sqrt{\operatorname{det} \gamma}}\right)+\nabla_{i}\left(\frac{R_{j}^{(g)}}{\sqrt{\operatorname{det} \gamma}}\right) D^{j}{ }_{k} n^{k}\right]\left(L n^{i}+L^{i}\right)\right)
\end{aligned}
$$

where

$$
U^{m n} \equiv \frac{2}{\sqrt{\operatorname{det} \gamma}} \frac{\delta(\sqrt{\operatorname{det} \gamma} U)}{\delta \gamma_{m n}}=U \gamma^{m n}+2 \frac{\partial U}{\partial \gamma_{m n}} .
$$

As for the \{\}$_{f}$ bracket, since $\mathcal{C}$ is independent of $p^{i j}$, the only nonzero contributions will come from the $p^{i j}$ dependent terms in $\tilde{R}^{0}$ and $\tilde{R}_{i}$, combined with $\phi_{i j}$ dependent terms in $\mathcal{C}$,

$$
\left\{\mathcal{C}(z), L^{i}(y) \tilde{R}_{i}(y)+L(y) \tilde{R}^{0}(y)\right\}_{f}=Z^{m n}(z)\left(L^{k}(y) \frac{\delta R_{k}^{(f)}(y)}{\delta p^{m n}(z)}+L(y) \frac{\delta R^{0(f)}(y)}{\delta p^{m n}(z)}\right)
$$


where $Z^{m n}$ is given by,

$$
Z^{m n}=\frac{\partial \mathcal{C}}{\partial \phi_{m n}}=R_{j}^{(g)} \frac{\partial\left(D^{j}{ }_{k} n^{k}\right)}{\partial \phi_{m n}}+2 m^{4} \sqrt{\operatorname{det} \gamma} \frac{\partial V}{\partial \phi_{m n}} .
$$

To compute $Z^{m n}$, we need the following expressions that can be derived from (2.16),

$$
\begin{aligned}
\frac{\partial}{\partial \phi_{m n}} \operatorname{Tr}(\sqrt{x} D) & =-\frac{1}{\sqrt{x}}\left[n^{i} \phi_{i j} \frac{\partial\left(D^{j}{ }_{k} n^{k}\right)}{\partial \phi_{m n}}-\frac{1}{2} Q_{1}^{m n}\right], \\
\frac{\partial}{\partial \phi_{m n}} \operatorname{Tr}\left(x D^{2}\right) & =-2\left[D^{i}{ }_{l} n^{l} \phi_{i j} \frac{\partial\left(D^{j}{ }_{k} n^{k}\right)}{\partial \phi_{m n}}-\frac{1}{2} Q_{2}^{m n}\right], \\
\frac{\partial}{\partial \phi_{m n}} \operatorname{Tr}\left(x^{3 / 2} D^{3}\right) & =-3 \sqrt{x}\left[D^{i}{ }_{l} D^{l}{ }_{r} n^{r} \phi_{i j} \frac{\partial\left(D^{j}{ }_{k} n^{k}\right)}{\partial \phi_{m n}}-\frac{1}{2} Q_{3}^{m n}\right],
\end{aligned}
$$

where $Q_{1}^{m n}, Q_{2}^{m n}$ and $Q_{3}^{m n}$ are defined as,

$$
\begin{aligned}
& Q_{1}^{m n}=\left(D^{-1}\right)^{m}{ }_{i} \gamma^{n i}-n^{i} n^{m} D^{n}{ }_{i}, \\
& Q_{2}^{m n}=\gamma^{m n}-D^{m}{ }_{i} n^{i} D^{n}{ }_{j} n^{j}, \\
& Q_{3}^{m n}=D^{m}{ }_{i} \gamma^{i n}-D^{m}{ }_{i} D^{i}{ }_{j} n^{j} D^{n}{ }_{k} n^{k},
\end{aligned}
$$

and can be shown to be symmetric using (2.16). Differentiating equation (2.25) with respect to $f_{m n}$, using (3.6)-(3.8) and performing some algebraic manipulations yields,

$$
\begin{aligned}
\frac{\partial V}{\partial \phi_{m n}}=-\frac{n^{l} \phi_{l k}}{\sqrt{x}}[ & \beta_{1} \delta^{k}{ }_{i}+\beta_{2} \sqrt{x}\left(\delta^{k}{ }_{i} D^{r}{ }_{r}-D^{k}{ }_{i}\right) \\
& \left.+\beta_{3} x\left(\delta^{k}{ }_{i} e_{2}(D)+D_{r}^{k}{ }_{r}{ }^{r}{ }_{i}-D^{k}{ }_{i} D_{r}^{r}\right)\right] \frac{\partial\left(D^{i}{ }_{j} n^{j}\right)}{\partial \phi_{m n}}+\frac{1}{2} \bar{W}^{m n}
\end{aligned}
$$

where

$$
\bar{W}^{m n}=\frac{\beta_{1}}{\sqrt{x}} Q_{1}^{m n}+\beta_{2}\left(D^{i}{ }_{i} Q_{1}^{m n}-Q_{2}^{m n}\right)+\beta_{3} \sqrt{x}\left(e_{2}(D) Q_{1}^{m n}-D^{i}{ }_{i} Q_{2}^{m n}+Q_{3}^{m n}\right) .
$$

On inserting (3.12) into (3.5) and imposing (2.30), $Z^{m n}$ becomes,

$$
Z^{m n}=m^{4} \sqrt{\operatorname{det} \gamma} \bar{W}^{m n} .
$$

Now, to integrate (3.4), we use

$$
\begin{aligned}
\frac{\delta R^{0(f)}(y)}{\delta p^{m n}(z)} & =\frac{1}{M_{f}^{2} \sqrt{\operatorname{det} \phi}}\left(\phi_{m n}(y) p_{k}^{k}(y)-2 p_{m n}(y)\right) \delta^{3}(z-y), \\
\int \mathrm{d}^{3} y L^{i}(y) \frac{\delta R_{i}^{(f)}(y)}{\delta p^{m n}(z)} & =-\left[\left(\bar{\nabla}_{z}\right)_{m} L_{n}(z)+\left(\bar{\nabla}_{z}\right)_{n} L_{m}(z)\right],
\end{aligned}
$$

which are similar to the corresponding expressions for $R^{0(g)}$ and $R_{i}^{(g)}$ in [4]. Performing the integration, and using equation (3.14), yields

$$
\begin{aligned}
-\int \mathrm{d}^{3} y & \left\{\mathcal{C}(z), L^{i}(y) \tilde{R}_{i}(y)+L(y) \tilde{R}^{0}(y)\right\}_{f} \\
= & m^{4} \sqrt{\operatorname{det} \gamma} \bar{W}^{m n}\left(\frac{2 L}{M_{f}^{2} \sqrt{\operatorname{det} \phi}}\left(p_{m n}-\frac{1}{2} \phi_{m n} p_{k}^{k}\right)+\bar{\nabla}_{m} L_{n}+\bar{\nabla}_{n} L_{m}\right) .
\end{aligned}
$$


Combining this with (3.2) gives a preliminary expression for the secondary constraint in bimetric theory which seemingly depends on $L, L_{i}$ and their spatial derivatives,

$$
\begin{aligned}
\mathcal{C}_{(2)} \approx & \frac{m^{4}}{M_{g}^{2}} L\left(\gamma_{m n} \pi_{k}^{k}-2 \pi_{m n}\right) U^{m n}+2 m^{4} \sqrt{\operatorname{det} \gamma} \gamma_{n i} D^{i}{ }_{k} n^{k} \nabla_{m}\left(L U^{m n}\right) \\
& +\left(R_{j}^{(g)} D^{i}{ }_{k} n^{k}-2 m^{4} \sqrt{\operatorname{det} \gamma} \gamma_{j k} \bar{V}^{k i}\right) \nabla_{i}\left(L n^{j}+L^{j}\right) \\
& +\sqrt{\operatorname{det} \gamma}\left[\nabla_{i}\left(\frac{R^{0(g)}}{\sqrt{\operatorname{det} \gamma}}\right)+\nabla_{i}\left(\frac{R_{j}^{(g)}}{\sqrt{\operatorname{det} \gamma}}\right) D^{j}{ }_{k} n^{k}\right]\left(L n^{i}+L^{i}\right) \\
& -\frac{m^{4}}{M_{f}^{2}} L \frac{\sqrt{\operatorname{det} \gamma}}{\sqrt{\operatorname{det} \phi}}\left(\phi_{m n} p_{k}^{k}-2 p_{m n}\right) \bar{W}^{m n}+2 m^{4} \sqrt{\operatorname{det} \gamma} \phi_{j k} \bar{W}^{k i} \bar{\nabla}_{i} L^{j}
\end{aligned}
$$

One can verify that $\mathcal{C}_{(2)}$ is consistent with the corresponding expression in massive gravity. For this, note that the last line of (3.18) coming from (3.17) is related to the $\phi_{m n}$ equation of motion,

$$
\frac{\partial}{\partial t} \phi_{m n}=\frac{2 L}{M_{f}^{2} \sqrt{\operatorname{det} \phi}}\left(p_{m n}-\frac{1}{2} \phi_{m n} p_{k}^{k}\right)+\bar{\nabla}_{m} L_{n}+\bar{\nabla}_{n} L_{m}
$$

Hence, using (3.5) and (3.14), we have,

$$
-\int \mathrm{d}^{3} y\left\{\mathcal{C}(z), L^{i}(y) \tilde{R}_{i}(y)+L(y) \tilde{R}^{0}(y)\right\}_{f}=\frac{\partial \mathcal{C}}{\partial \phi_{m n}} \frac{\partial}{\partial t} \phi_{m n}
$$

In massive gravity where $\phi_{m n}$ is nondynamical, the right hand side corresponds to the explicit time dependence of $\mathcal{C}$ through the $f_{\mu \nu}$ background. More precisely, consider,

$$
\mathcal{C}_{(2)}=\frac{d}{d t} \mathcal{C}=\frac{\partial \mathcal{C}}{\partial \gamma_{i j}} \frac{\partial \gamma_{i j}}{\partial t}+\frac{\partial \mathcal{C}}{\partial \pi^{i j}} \frac{\partial \pi^{i j}}{\partial t}+\frac{\partial \mathcal{C}}{\partial \phi_{i j}} \frac{\partial \phi_{i j}}{\partial t}+\frac{\partial \mathcal{C}}{\partial p^{i j}} \frac{\partial p^{i j}}{\partial t}
$$

The last term vanishes since $\mathcal{C}$ does not depend on $p^{i j}$, and the third term is the one in (3.20). The first two terms produce the Poisson bracket with respect to $\left(\gamma_{i j}, \pi^{i j}\right)$ and are common with massive gravity. Then, from (3.18), the expression for $\mathcal{C}_{(2)}$ in massive gravity becomes,

$$
\begin{aligned}
\mathcal{C}_{(2)}^{\mathrm{MG}} \approx & \frac{\partial \mathcal{C}}{\partial \phi_{i j}} \frac{\partial \phi_{i j}}{\partial t}+\frac{m^{4}}{M_{g}^{2}} L\left(\gamma_{m n} \pi^{k}{ }_{k}-2 \pi_{m n}\right) U^{m n}+2 m^{4} \sqrt{\operatorname{det} \gamma} \gamma_{n i} D^{i}{ }_{k} n^{k} \nabla_{m}\left(L U^{m n}\right) \\
& +\left(R_{j}^{(g)} D^{i}{ }_{k} n^{k}-2 m^{4} \sqrt{\operatorname{det} \gamma} \gamma_{j k} \bar{V}^{k i}\right) \nabla_{i}\left(L n^{j}+L^{j}\right) \\
& +\sqrt{\operatorname{det} \gamma}\left[\nabla_{i}\left(\frac{R^{0(g)}}{\sqrt{\operatorname{det} \gamma}}\right)+\nabla_{i}\left(\frac{R_{j}^{(g)}}{\sqrt{\operatorname{det} \gamma}}\right) D^{j}{ }_{k} n^{k}\right]\left(L n^{i}+L^{i}\right)
\end{aligned}
$$

which is consistent with the expression in [4]. ${ }^{6}$

\footnotetext{
${ }^{6}$ In [4], the $L$ in the third term of (3.22) is outside the covariant derivative which is a misprint corrected here. Also the first term is absent for backgrounds with $f_{\mu \nu}=\eta_{\mu \nu}$.
} 


\subsection{The $L^{i}$ independence of $\mathcal{C}_{(2)}$}

Note that all $L^{i}$ dependent terms of $\mathcal{C}_{(2)}$ arise from the bracket $\left\{\mathcal{C}(z), L^{i}(y) \tilde{R}_{i}(y)\right\}$ in equation (3.1). In this section, we show that, on the constraint surface, all such terms vanish in bimetric theory (though not in massive gravity). Denoting the $L^{i}$ dependent part of $\mathcal{C}_{(2)}$ in (3.18) by $\mathcal{C}_{S}$, one gets,

$$
\begin{aligned}
\mathcal{C}_{S} \approx & \sqrt{\operatorname{det} \gamma}\left[\nabla_{i}\left(\frac{R^{0(g)}}{\sqrt{\operatorname{det} \gamma}}\right)+\nabla_{i}\left(\frac{R_{j}^{(g)}}{\sqrt{\operatorname{det} \gamma}}\right) D^{j}{ }_{k} n^{k}\right] L^{i} \\
& +R_{j}^{(g)} D^{i}{ }_{k} n^{k} \nabla_{i} L^{j}-2 m^{4} \sqrt{\operatorname{det} \gamma}\left(\gamma_{j k} \bar{V}^{k i} \nabla_{i} L^{j}-\phi_{j k} \bar{W}^{k i} \bar{\nabla}_{i} L^{j}\right) .
\end{aligned}
$$

By adding and subtracting terms, the first line can be completed to include $\mathcal{C}(2.22)$, and we get,

$$
\mathcal{C}_{S} \approx L^{i} \partial_{i} \mathcal{C}-\Delta
$$

To write the first term, we have used the fact that $\mathcal{C}$ is a scalar density so that $\mathcal{C} / \sqrt{\operatorname{det} \gamma}$ is a scalar. Hence, one has $\sqrt{\operatorname{det} \gamma} \nabla_{i}(\mathcal{C} / \sqrt{\operatorname{det} \gamma}) \approx \partial_{i} \mathcal{C}$, on the constraint surface. Since $\mathcal{C} \approx 0$ at all points on a spacelike hypersurface, it follows that $\partial_{i} \mathcal{C} \approx 0$. In the second term, the quantity $\Delta$ stands for,

$$
\begin{aligned}
\Delta= & R_{j}^{(g)}\left(\nabla_{i}\left(D^{j}{ }_{k} n^{k}\right) L^{i}-D^{i}{ }_{k} n^{k} \nabla_{i} L^{j}\right) \\
& +2 m^{4} \sqrt{\operatorname{det} \gamma}\left(\partial_{i} V L^{i}+\gamma_{j k} \bar{V}^{k i} \nabla_{i} L^{j}-\phi_{j k} \bar{W}^{k i} \bar{\nabla}_{i} L^{j}\right) .
\end{aligned}
$$

Since $\int \mathrm{d}^{3} y\left\{\mathcal{C}(z), L^{i}(y) \tilde{R}_{i}(y)\right\}=-\mathcal{C}_{S}(z)$, the quantity $\Delta$ appears in the computation of this bracket in appendix B.3, more precisely, in equation (B.88). A lengthy calculation, which is relegated to the appendix, shows that $\Delta=0$ and therefore, $\mathcal{C}_{S} \approx 0$. The dependence on $L^{i}$ therefore vanishes entirely and all such terms should be dropped from the expression for $\mathcal{C}_{(2)}$ in $(3.18)$.

\subsection{The $\partial_{i} L$ independence of $\mathcal{C}_{(2)}$}

In this section, it is shown that all terms in $\mathcal{C}_{(2)}$ involving derivatives of $L$ vanish on the constraint surface. Denote the $\partial_{i} L$ dependent part of $\mathcal{C}_{(2)}$ by $\mathcal{C}_{D}$. From equation (3.18) it follows that

$$
\begin{aligned}
\mathcal{C}_{D}= & \left(R_{j}^{(g)} n^{j}+2 m^{4} \sqrt{\operatorname{det} \gamma} U\right) D^{i}{ }_{k} n^{k} \partial_{i} L \\
& +4 m^{4} \sqrt{\operatorname{det} \gamma} \gamma_{n i} D^{i}{ }_{k} n^{k} \frac{\partial U}{\partial \gamma_{m n}} \partial_{m} L-2 m^{4} \sqrt{\operatorname{det} \gamma} \gamma_{i n} \bar{V}^{n m} n^{i} \partial_{m} L
\end{aligned}
$$

where we have used the expression (3.3) for $U^{m n}$. The first two terms are simplified by using (2.24) and (2.17) to rewrite $U$, and then imposing (2.30) to get,

$$
\left(R_{j}^{(g)} n^{j}+2 m^{4} \sqrt{\operatorname{det} \gamma} U\right)=2 m^{4} \sqrt{\operatorname{det} \gamma}\left[\frac{\beta_{1}}{\sqrt{x}}+\beta_{2} D^{l}{ }_{l}+\beta_{3} \sqrt{x} e_{2}(D)\right] .
$$


Note that $U$ given in (2.24) has the form $U=\beta_{1}\left[U_{1}\right]+\beta_{2}\left[U_{2}\right]+\beta_{3}\left[U_{3}\right]$. To simplify the third term in $\mathcal{C}_{D}$, we need to compute $\frac{\partial U}{\partial \gamma_{m n}}$ (at fixed $n_{i}$ ). The $\beta_{1}$-term vanishes trivially,

$$
\beta_{1} \frac{\partial\left[U_{1}\right]}{\partial \gamma_{m n}}=\beta_{1} \frac{\partial \sqrt{x}}{\partial \gamma_{m n}}=0 .
$$

The $\beta_{2}$-term is also easily evaluated using the first equation in (2.38),

$$
\beta_{2} \frac{\partial\left[U_{2}\right]}{\partial \gamma_{m n}}=\frac{\beta_{2}}{2} \phi_{i j}\left(D^{-1}\right)^{j}{ }_{k} \frac{\partial \gamma^{k i}}{\partial \gamma_{m n}} .
$$

Similarly, using (2.38), the $\beta_{3}$-term becomes,

$$
\beta_{3} \frac{\partial\left[U_{3}\right]}{\partial \gamma_{m n}}=\frac{\beta_{3}}{2 \sqrt{x}}\left[x D_{l}^{l}{ }_{l}\left(D^{-1}\right)_{k}^{j}-x \delta_{k}^{j}+n^{l} \phi_{l q} D^{q}{ }_{r} n^{r}\left(D^{-1}\right)_{k}^{j}-n^{l} \phi_{l k} n^{j}\right] \phi_{j i} \frac{\partial \gamma^{k i}}{\partial \gamma_{m n}},
$$

where we have also used the relation, ${ }^{7}$

$$
n^{l} \phi_{l i}\left(x D^{i}{ }_{j}+D^{i}{ }_{r} n^{r} n^{q} \phi_{q j}\right) \frac{\partial\left(D^{j}{ }_{k} n^{k}\right)}{\partial \gamma_{m n}}=\frac{1}{2} n^{i} \phi_{i j} n^{l} \phi_{l k} \frac{\partial \gamma^{j k}}{\partial \gamma_{m n}} .
$$

Now, substituting (3.28), (3.29), (3.30) and (3.31) in the expression for $\mathcal{C}_{D}$, one can see that it vanishes on using the identity,

$$
D_{k}^{i} n^{k}-n^{j} \phi_{j m}\left(D^{-1}\right)^{m}{ }_{n} \gamma^{n i}=0,
$$

which follows from equations (2.16) and (2.17) that define the matrix $D$. For example, the $\beta_{1}$-term of $\mathcal{C}_{D}$ is given by,

$$
\mathcal{C}_{D}^{\beta_{1}}=2 m^{4} \sqrt{\operatorname{det} \gamma} \frac{\beta_{1}}{\sqrt{x}}\left[D^{i}{ }_{k} n^{k}-n^{j} \phi_{j m}\left(D^{-1}\right)^{m}{ }_{n} \gamma^{n i}\right] \partial_{i} L=0 .
$$

Similarly one can show that $\mathcal{C}_{D}^{\beta_{2}}$ and $\mathcal{C}_{D}^{\beta_{3}}$ also vanish on repeated application of (3.33), hence, $\mathcal{C}_{D}=0$. This means that $\mathcal{C}_{(2)}$ is independent of $\partial_{i} L$ and can be expressed as,

$$
\begin{aligned}
\mathcal{C}_{(2)} \approx & L\left(\frac{m^{4}}{M_{g}^{2}}\left(\gamma_{m n} \pi^{k}{ }_{k}-2 \pi_{m n}\right) U^{m n}+2 m^{4} \sqrt{\operatorname{det} \gamma} \gamma_{n i} D^{i}{ }_{k} n^{k} \nabla_{m} U^{m n}\right. \\
& +\left(R_{j}^{(g)} D^{i}{ }_{k} n^{k}-2 m^{4} \sqrt{\operatorname{det} \gamma} \gamma_{j k} \bar{V}^{k i}\right) \nabla_{i} n^{j} \\
& +\sqrt{\operatorname{det} \gamma}\left[\nabla_{i}\left(\frac{R^{0(g)}}{\sqrt{\operatorname{det} \gamma}}\right)+\nabla_{i}\left(\frac{R_{j}^{(g)}}{\sqrt{\operatorname{det} \gamma}}\right) D^{j}{ }_{k} n^{k}\right] n^{i} \\
& \left.-\frac{m^{4}}{M_{f}^{2}} \frac{\sqrt{\operatorname{det} \gamma}}{\sqrt{\operatorname{det} \phi}}\left(\phi_{m n} p_{k}^{k}-2 p_{m n}\right) \bar{W}^{m n}\right) .
\end{aligned}
$$

Since $L$ is an overall factor, imposing this secondary constraint is equivalent to $\mathcal{C}_{(2)} / L \approx 0$. This can be solved for the canonical momentum of the ghost mode.

One can check that there are no additional secondary constraints. From (4.4) and (4.5) below, we see that the brackets $\left\{\tilde{R}^{0}(x), \tilde{R}_{i}(y)\right\}$ and $\left\{\tilde{R}^{0}(x), \tilde{R}^{0}(y)\right\}$ vanish on the constraint surface. Thus, the condition $\dot{\tilde{R}}^{0} \approx 0$, leads to an equation that is identical with (3.35), (except that $L$ is replaced by $N$ ) and imposes the same constraint. This, combined with the fact that $\dot{\tilde{R}}_{i} \approx 0$ is automatically satisfied, shows that there are no further constraints.

\footnotetext{
${ }^{7}$ Equation (3.32) is analogous to equation (4.6) in [10], and is derived in a similar way.
} 


\section{The algebra of general covariance and the HKT metric}

\subsection{The algebra of general covariance}

In general relativity, the Poisson brackets of the first class constraints, $R_{0} \approx 0, R_{i} \approx 0$, are [41],

$$
\begin{aligned}
& \left\{R^{0}(x), R^{0}(y)\right\}=R^{i}(y) \frac{\partial}{\partial y^{i}} \delta^{3}(x-y)-R^{i}(x) \frac{\partial}{\partial x^{i}} \delta^{3}(x-y), \\
& \left\{R^{0}(x), R_{i}(y)\right\}=-R^{0}(y) \frac{\partial}{\partial x^{i}} \delta^{3}(x-y), \\
& \left\{R_{i}(x), R_{j}(y)\right\}=R_{i}(y) \frac{\partial}{\partial y^{j}} \delta^{3}(x-y)-R_{j}(x) \frac{\partial}{\partial x^{i}} \delta^{3}(x-y) .
\end{aligned}
$$

This is the algebra of spacetime diffeomorphisms and is not unique to general relativity. Any covariant field theory will have first class constraints satisfying the algebra (4.1) after second class constraints have been imposed [30]. This is a necessary and sufficient condition for the constraints to generate spacetime diffeomorphisms.

Since the algebra (4.1) contains both $R_{i}$ and $R^{i}$, it implicitly contains a metric to manipulate the index. In general relativity and theories minimally coupled to it, this is the spatial metric $\gamma_{i j} \equiv g_{i j}$. Furthermore, in such theories, the diffeomorphism constraints appear in the Hamiltonian with Lagrange multipliers, as

$$
\mathcal{H}=-N R^{0}-N^{i} R_{i}
$$

provided second class constraints and their stability conditions, if any, are already imposed. Here, $N$ and $N^{i}$ are the lapse and shift functions that complete $g_{i j}$ to $g_{\mu \nu}$.

This observations can help identify a spacetime metric in a diffeomorphism invariant theory. The algebra (4.1) also holds in generally covariant theories where a spacetime metric is not a priori specified. Then, as conjectured by Hojman, Kuchař and Teitelboim [5], the tensor that relates $R_{i}$ and $R^{i}$ can be identified as the metric of spacelike hypersurfaces. An explicit example of this, for Ashtekar's canonical formulation of gravity, is given in [42]. Here we are interested in the implications of the conjecture for bimetric theory which does not a priori have a unique metric candidate. We will obtain the algebra of first class constraints and show that it leads to different HKT metrics depending on how the constraints are identified. Hence in these type of models the HKT conjecture does not lead to a unique metric.

\subsection{First class constraints in bimetric theory}

Bimetric theory has six independent constraints, $\tilde{R}^{0}, \tilde{R}_{i}, \mathcal{C}$ and $\mathcal{C}_{(2)}$, given by equations (2.20)-(2.22) and (3.35). Four linear combinations of these should be first class and satisfy the algebra (4.1). We first show that $\tilde{R}_{i}$ are first class. The Poisson brackets 
of the first five constraints are

$$
\begin{aligned}
\left\{\tilde{R}_{i}(x), \tilde{R}_{j}(y)\right\} & =-\left[\tilde{R}_{j}(x) \frac{\partial}{\partial x^{i}} \delta^{3}(x-y)-\tilde{R}_{i}(y) \frac{\partial}{\partial y^{j}} \delta^{3}(x-y)\right], \\
\left\{\tilde{R}^{0}(x), \tilde{R}_{i}(y)\right\} & =-\tilde{R}^{0}(y) \frac{\partial}{\partial x^{i}} \delta^{3}(x-y), \\
\left\{\tilde{R}^{0}(x), \tilde{R}^{0}(y)\right\} & =-\left[\phi^{i j}(x) \tilde{R}_{j}(x) \frac{\partial}{\partial x^{i}} \delta^{3}(x-y)-\phi^{i j}(y) \tilde{R}_{j}(y) \frac{\partial}{\partial y^{i}} \delta^{3}(x-y)\right], \\
\left\{\mathcal{C}(x), \tilde{R}_{i}(y)\right\} & =-\mathcal{C}(y) \frac{\partial}{\partial x^{i}} \delta^{3}(x-y), \\
\{\mathcal{C}(x), \mathcal{C}(y)\} & =-\left[\mathcal{C}(x) D^{i}{ }_{j} n^{j}(x) \frac{\partial}{\partial x^{i}} \delta^{3}(x-y)-\mathcal{C}(y) D^{i}{ }_{j} n^{j}(y) \frac{\partial}{\partial y^{i}} \delta^{3}(x-y)\right], \\
\left\{\mathcal{C}(x), \tilde{R}^{0}(y)\right\} & =-\frac{\mathcal{C}(2)(x)}{L(x)} \delta^{3}(x-y) .
\end{aligned}
$$

Equation (4.3) is easily derived from (2.21), and the fact that $R_{i}^{(g)}$ and $R_{i}^{(f)}$ each satisfy the spatial part of (4.1). The bracket (4.7) is given in (2.41) and the remaining ones are derived in appendix B. All of these brackets vanish on the constraint surface.

Now consider the brackets involving $\mathcal{C}_{(2)}$, starting with $\left\{\mathcal{C}_{(2)}(x), \tilde{R}_{i}(y)\right\}$. This vanishes on the constraint surface as seen from the definition of $\mathcal{C}_{(2)}$ in (2.32) and the Jacobi identity,

$$
\left\{\mathcal{C}_{(2)}(x), \tilde{R}_{i}(y)\right\}=\left\{\{\mathcal{C}(x), H\}, \tilde{R}_{i}(y)\right\}=\left\{\mathcal{C}(x),\left\{H, \tilde{R}_{i}(y)\right\}\right\}-\left\{H,\left\{\mathcal{C}(x), \tilde{R}_{i}(y)\right\}\right\} .
$$

Indeed, using (4.6), the last bracket becomes,

$$
\left\{H,\left\{\mathcal{C}(x), \tilde{R}_{i}(y)\right\}\right\}=\mathcal{C}_{(2)}(y) \frac{\partial}{\partial x^{i}} \delta^{3}(x-y),
$$

which is weakly zero. In addition, using (2.33), as well as (4.3), (4.4) and (4.6) it follows that

$$
\left\{H, \tilde{R}_{i}(y)\right\}=-\left[\frac{\partial N(y)}{\partial y^{i}} \mathcal{C}(y)+\frac{\partial L(y)}{\partial y^{i}} \tilde{R}^{0}(y)+\frac{\partial}{\partial y^{j}}\left(L^{j}(y) \tilde{R}_{i}(y)\right)+\frac{\partial L^{j}(y)}{\partial y^{i}} \tilde{R}_{j}(y)\right] .
$$

Now all brackets in $\left\{\mathcal{C}(x),\left\{H, \tilde{R}_{i}(y)\right\}\right\}$ are known and result in terms proportional to a constraint or its spatial derivative. Hence, this expression, and therefore $\left\{\mathcal{C}_{(2)}(x), \tilde{R}_{i}(y)\right\}$, is weakly zero, showing that the Poisson brackets of $\tilde{R}_{i}$ with all constraints vanish weakly. Therefore, $\tilde{R}_{i}$ are first class constraints generating the algebra of spatial diffeomorphisms in (4.1).

To find the remaining first class constraint, we turn our attention to the brackets $\left\{\mathcal{C}(x), \mathcal{C}_{(2)}(y)\right\}$ and $\left\{\tilde{R}^{0}(x), \mathcal{C}_{(2)}(y)\right\}$, neither of which is weakly zero. This can be seen by noting that they appear in the expression for $\dot{\mathcal{C}}_{(2)}$, which, on using $(2.33)$ and $\left\{\mathcal{C}_{(2)}(x), \tilde{R}_{i}(y)\right\} \approx 0$, becomes,

$$
\dot{\mathcal{C}}_{(2)}(x) \approx-\int \mathrm{d} y\left[L(y)\left\{\mathcal{C}_{(2)}(x), \tilde{R}^{0}(y)\right\}+N(y)\left\{\mathcal{C}_{(2)}(x), \mathcal{C}(y)\right\}\right] .
$$

This is computed at the linear level in appendix A and the result, in equation (A.45), shows that the two brackets are not weakly zero. Hence, none of the constraints $\tilde{R}^{0}, \mathcal{C}$ or $\mathcal{C}_{(2)}$ is first class. 
However, a first class combination of them could exist [40]. Note that the brackets $\left\{\mathcal{C}(x), \mathcal{C}_{(2)}(y)\right\}$ and $\left\{\tilde{R}^{0}(x), \mathcal{C}_{(2)}(y)\right\}$ contain no derivatives of delta functions, as can be shown using the Jacobi identity, similar to the analysis in [22]. Then they have the form,

$$
\begin{aligned}
\left\{\mathcal{C}(x), \mathcal{C}_{(2)}(y)\right\} & =L(x) D(x) \delta^{3}(x-y), \\
\left\{\tilde{R}^{0}(x), \mathcal{C}_{(2)}(y)\right\} & =L(x) E(x) \delta^{3}(x-y),
\end{aligned}
$$

where $D$ and $E$ are nonzero functions of the phase space variables. Now define the constraint $\hat{R}^{0}$ as the linear combination,

$$
\hat{R}^{0} \equiv W \mathcal{C}+\tilde{R}^{0}, \quad W \equiv-\frac{E}{D}
$$

The Poisson bracket of $\hat{R}^{0}$ and $\mathcal{C}_{(2)}$ is now weakly zero,

$$
\begin{aligned}
\left\{\hat{R}^{0}(x), \mathcal{C}_{(2)}(y)\right\} & =\left\{W(x), \mathcal{C}_{(2)}(y)\right\} \mathcal{C}(x)+W(x)\left\{\mathcal{C}(x), \mathcal{C}_{(2)}(y)\right\}+\left\{\tilde{R}^{0}(x), \mathcal{C}_{(2)}(y)\right\} \\
& \approx-\frac{E(x)}{D(x)} L(x) D(x) \delta^{3}(x-y)+L(x) E(x) \delta^{3}(x-y)=0 .
\end{aligned}
$$

Moreover, from (4.4)-(4.8), it follows that the brackets of $\hat{R}^{0}$ with other constraints also vanish on the constraint surface. Hence, $\hat{R}^{0}$ is a first class constraint. Note that we will find the same constraint by solving the stability condition $\dot{\mathcal{C}}_{(2)} \approx 0$ for $N$ and replacing it in the Hamiltonian. Indeed, using (4.13) and (4.14) in (4.12) and setting $\dot{\mathcal{C}}_{(2)} \approx 0$ gives $N=W L$. This, when inserted into the Hamiltonian (2.33), reduces it to

$$
\mathcal{H}=-L \hat{R}^{0}-L^{i} \tilde{R}_{i}
$$

which is the desired form (4.2). Furthermore, using (4.4)-(4.8), it follows that

$$
\begin{aligned}
&\left\{\hat{R}^{0}(x), \tilde{R}_{i}(y)\right\} \approx-\hat{R}^{0}(y) \frac{\partial}{\partial x^{i}} \delta^{3}(x-y), \\
&\left\{\hat{R}^{0}(x), \hat{R}^{0}(y)\right\} \approx-\left[\phi^{i j}(x) \tilde{R}_{j}(x) \frac{\partial}{\partial x^{i}} \delta^{3}(x-y)-\phi^{i j}(y) \tilde{R}_{j}(y) \frac{\partial}{\partial y^{i}} \delta^{3}(x-y)\right] .
\end{aligned}
$$

The weak equalities $\approx$ now hold on the surface of the second class constraints $\mathcal{C}$ and $\mathcal{C}_{(2)} . \quad \hat{R}^{0}$ generates time diffeomorphisms as in (4.1). $\hat{R}^{0}$ and $\tilde{R}_{i}$ together generate the diffeomorphism algebra.

\subsection{The HKT metrics}

It is obvious that on the right hand side of (4.19), the index of $\tilde{R}_{i}$ is raised using $\phi^{i j}$, hence, by the HKT conjecture [5] described above, $f_{i j} \equiv \phi_{i j}$ will be a HKT spatial metric in bimetric theory. Furthermore, the Lagrange multipliers $L$ and $L^{i}$ in the Hamiltonian (4.17) will be the corresponding lapse and shift functions, leading to a spacetime metric $f_{\mu \nu}$.

Singling out $f_{\mu \nu}$ in this way might seem odd considering that both metrics appear in the bimetric action on similar footing. But this is a consequence of the choice of variables that was made to arrive at the constraints. Obviously, it is possible to repeat all manipulations with $g_{\mu \nu}$ and $f_{\mu \nu}$ interchanged, as commented in footnote 3 . This will result in a 
Lagrangian linear in $L, N$ and $N^{i}$, and finally $\gamma_{i j}$ will appear as the HKT metric in the covariance algebra.

In fact, it is not even necessary to repeat all steps with the $g$ and $f$ metrics interchanged. We can start with the $n^{i}$ still given by (2.15) but use them to eliminate the $L^{i}$ (instead of the $N^{i}$ ). The resulting Lagrangian has the form,

$$
\mathcal{L}=\pi^{i j} \partial_{t} \gamma_{i j}+p^{i j} \partial_{t} \phi_{i j}+N^{i} \tilde{R}_{i}+L \mathcal{C}_{L}+N \mathcal{C}_{N}
$$

where $\mathcal{C}_{N}$ and $\mathcal{C}_{L}$ differ from $\mathcal{C}$ and $\tilde{R}^{0}$. Then, proceeding as in the previous section, the stability conditions of $\mathcal{C}_{N}$ and $\mathcal{C}_{L}$ provide a sixth constraint, whose stability condition in turn can be solved for $L$. Using this solution gives a Hamiltonian $\mathcal{H}=-N \tilde{\tilde{R}}^{0}-N^{i} \tilde{R}_{i}$, where $\tilde{\tilde{R}}^{0}$ and $\tilde{R}_{i}$ are first class constraints satisfying the spatial diffeomorphism algebra and,

$$
\left\{\tilde{\tilde{R}}^{0}(x), \tilde{\tilde{R}}^{0}(y)\right\} \approx-\left[\gamma^{i j}(x) \tilde{R}_{j}(x) \frac{\partial}{\partial x^{i}} \delta^{3}(x-y)-\gamma^{i j}(y) \tilde{R}_{j}(y) \frac{\partial}{\partial y^{i}} \delta^{3}(x-y)\right],
$$

which yields $\gamma_{i j}$ as the spatial HKT metric and $g_{\mu \nu}$ as the spacetime metric. Hence, both $g_{\mu \nu}$ and $f_{\mu \nu}$ can arise as HKT metrics, consistent with the general structure of the theory. ${ }^{8}$ This is also consistent with the nature of ghost free matter couplings, whereby, a certain type of matter can only couple minimally to one of the metrics.

The nonuniqueness of this metric is easy to understand. The observation made by HKT was that whenever the Hamiltonian can be expressed as $\mathcal{H}=-M \tilde{R}^{0}-M^{i} \tilde{R}_{i}$, with first class constraints $\tilde{R}^{0}$ and $\tilde{R}_{i}$ satisfying the covariance algebra, then a metric can be read off from the $\left\{\tilde{R}^{0}(x), \tilde{R}^{0}(y)\right\}$ bracket as described above. However, in a theory where diffeomorphisms act on different sectors, there may exist multiple ways of selecting the first class constraints (and the Lagrange multipliers) each leading to a different choice of the HKT metric. In bimetric theory there is a further restriction. If, for some choice of variables, the HKT metric involves a combination of $g$ and $f$ metrics, then that combination must transform as a rank-2 tensor whenever $g$ and $f$ are transformed under coordinate transformations. For example, if we follow the steps leading to the Hamiltonian (4.17), but then use $N=W L$ to eliminate $L$ in $(2.33)$, we obtain (with $\hat{\hat{R}}^{0}=\hat{R}^{0} / W$ ),

$$
\mathcal{H}=-N \hat{\hat{R}}^{0}-L^{i} \tilde{R}_{i}
$$

However, a covariant metric with lapse $N$ and shift $L^{i}$ does not exist. Indeed, the algebra of $\hat{\hat{R}}^{0}$ and $\tilde{R}_{i}$ is not of the canonical form (4.1). Two different redefinitions of the $\hat{\hat{R}}^{0}$ that restores the canonical form of the algebra will lead to the HKT metric being $f_{\mu \nu}$ or $g_{\mu \nu}$.

It may be possible to choose other variables than the ones described above. For example one may use any linear combination of the shifts, $M^{i}=a N^{i}+b L^{i}$ (with $a \neq-b$ ) as Lagrange multipliers. Using stability conditions of the constraints one would then put the Hamiltonian in the form

$$
\mathcal{H}=-M^{\mu} R_{\mu} .
$$

\footnotetext{
${ }^{8}$ What determines the metric is not so much the field redefinition (2.15), but rather what shifts are retained as Lagrange multipliers. If $N^{i}$ are retained the HKT-metric is $g_{\mu \nu}$, whereas $L^{i}$ lead to $f_{\mu \nu}$.
} 
If the $R_{\mu}$ algebra has the canonical form, it will be possible to identify a new HKT metric with $M^{0}$ and $M^{i}$ as the lapse and shift. This metric may be a complicated function of the phase space variables that depends on $a$ and $b$. The metric identified in [22] could be an example of this.

Note that, in any case, the physical gravitational metric is the one that minimally couples to matter, say $g_{\mu \nu}$, as dictated by absence of the ghost [2].

\section{Acknowledgments}

It is a pleasure to thank Ingemar Bengtsson and Mikica Kocic for fruitful discussions. This work was supported by a grant from the Swedish Research Council.

\section{A Determining the Lagrange multipliers}

In massive gravity, the requirement that $\mathcal{C}_{(2)} \approx 0$ is preserved under time evolution, gives an equation that determines the Lagrange multiplier $N$ [4]. In bimetric gravity, the corresponding equation depends on all five Lagrange multipliers $N, L$, and $L_{i}$,

$$
\begin{aligned}
\frac{\mathrm{d}}{\mathrm{d} t} \mathcal{C}_{(2)}(x) & =\left\{\mathcal{C}_{(2)}(x), H\right\} \\
& =-\int \mathrm{d} y\left[L^{i}(y)\left\{\mathcal{C}_{(2)}(x), \tilde{R}_{i}(y)\right\}+L(y)\left\{\mathcal{C}_{(2)}(x), \tilde{R}^{0}(y)\right\}+N(y)\left\{\mathcal{C}_{(2)}(x), \mathcal{C}(y)\right\}\right] \approx 0
\end{aligned}
$$

However, now four of the multipliers are determined by gauge fixing general coordinate transformations, analogous to the procedure in [38]. Then (A.1) becomes an equation for the fifth multiplier.

Here, we demonstrate this procedure explicitly in the linearized $\beta_{1}$-model: using general covariance to determine $L$ and $L^{i}$, (A.1) becomes an equation for $N$. We start with the equations of motion for $\gamma_{i j}, \phi_{i j}, \pi^{i j}$, and $p^{i j}$. Since the interaction terms in the action (2.1) do not depend on $\pi^{i j}$, varying with respect to it gives the same equation for $\gamma_{i j}$ as in general relativity,

$$
\partial_{t} \gamma_{i j}=\frac{2 N}{M_{g}^{2} \sqrt{\operatorname{det} \gamma}}\left(\pi_{i j}-\frac{1}{2} \gamma_{i j} \pi\right)+\nabla_{i} N_{j}+\nabla_{j} N_{i}
$$

where, $N_{i}$ are given by equation (2.15). Similarly, varying with respect to $p^{i j}$ leads to equation (3.19) for $\phi_{i j}$. However, since the interaction terms of (2.1) depend on $\gamma_{i j}$, the equation for $\pi^{i j}$ will have extra terms compared to the corresponding equation in general relativity. The extra terms can be computed using (2.37), (2.39) and (3.3), and the equation becomes,

$$
\begin{aligned}
\partial_{t} \pi^{i j}= & -M_{g}^{2} N \sqrt{\operatorname{det} \gamma}\left({ }^{3} R^{i j(g)}-\frac{1}{2} \gamma^{i j} R^{(g)}\right)+\frac{N}{2 M_{g}^{2} \sqrt{\operatorname{det} \gamma}} \gamma^{i j}\left(\pi^{m n} \pi_{m n}-\frac{1}{2} \pi^{2}\right) \\
& -\frac{2 N}{M_{g}^{2} \sqrt{\operatorname{det} \gamma}}\left(\pi^{i k} \pi_{k}^{j}-\frac{1}{2} \pi \pi^{i j}\right)+M_{g}^{2} \sqrt{\operatorname{det} \gamma}\left(\nabla^{i} \nabla^{j} N-\gamma^{i j} \nabla^{k} \nabla_{k} N\right) \\
& +\sqrt{\operatorname{det} \gamma}\left[\nabla_{k}\left(\frac{N^{k} \pi^{i j}}{\sqrt{\operatorname{det} \gamma}}\right)-\nabla_{k}\left(\frac{N^{i} \pi^{j k}}{\sqrt{\operatorname{det} \gamma}}\right)-\nabla_{k}\left(\frac{N^{j} \pi^{i k}}{\sqrt{\operatorname{det} \gamma}}\right)\right] \\
& +m^{4} \sqrt{\operatorname{det} \gamma}\left[N\left(V \gamma^{i j}-\bar{V}^{i j}\right)+L U^{i j}\right] .
\end{aligned}
$$


In the above, we express the $N^{i}$ in terms of $n^{i}$ given by equation (2.15). Analogously, using equations (3.5) and (3.14), the equation for $p^{i j}$ is (for general $\beta_{n}$ ),

$$
\begin{aligned}
\partial_{t} p^{i j}= & -M_{f}^{2} L \sqrt{\operatorname{det} \phi}\left({ }^{3} R^{i j(f)}-\frac{1}{2} \phi^{i j} R^{(f)}\right)+\frac{L}{2 M_{f}^{2} \sqrt{\operatorname{det} \phi}} \phi^{i j}\left(p^{m n} p_{m n}-\frac{1}{2} p^{2}\right) \\
& -\frac{2 L}{M_{f}^{2} \sqrt{\operatorname{det} \phi}}\left(p^{i k} p_{k}^{j}-\frac{1}{2} p p^{i j}\right)+M_{f}^{2} \sqrt{\operatorname{det} \phi}\left(\bar{\nabla}^{i} \bar{\nabla}^{j} L-\phi^{i j} \bar{\nabla}^{k} \bar{\nabla}_{k} L\right) \\
& +\sqrt{\operatorname{det} \phi}\left[\bar{\nabla}_{k}\left(\frac{L^{k} p^{i j}}{\sqrt{\operatorname{det} \phi}}\right)-\bar{\nabla}_{k}\left(\frac{L^{i} p^{j k}}{\sqrt{\operatorname{det} \phi}}\right)-\bar{\nabla}_{k}\left(\frac{L^{j} p^{i k}}{\sqrt{\operatorname{det} \phi}}\right)\right] \\
& +m^{4} \sqrt{\operatorname{det} \gamma}\left(N \bar{W}^{i j}+L \tilde{U}^{i j}\right) .
\end{aligned}
$$

Here, in analogy with (3.3), $\tilde{U}^{m n}$ is defined as

$$
\tilde{U}^{m n} \equiv 2 \frac{\partial U^{\prime}}{\partial \phi_{m n}}
$$

We now focus on the $\beta_{1}$-model by setting $\beta_{2}=\beta_{3}=0$. The equations will admit flat background solutions $g_{\mu \nu}=f_{\mu \nu}=\eta_{\mu \nu}$ if $\beta_{0}=-3 \beta_{1}$, and $\beta_{4}=-\beta_{1}$. which we also assume [31]. To linearize the theory around the flat background, we consider the small perturbations,

$$
N=1+\delta N, \quad \gamma_{i j}=\delta_{i j}+\hat{\gamma}_{i j}, \quad L=1+\delta L, \quad L_{i}=\delta L_{i}, \quad \phi_{i j}=\delta_{i j}+\hat{\phi}_{i j},
$$

with the boundary condition that all the perturbations vanish at infinity. A symmetric tensor perturbation admits the following linear orthogonal decomposition [38],

$$
a_{i j}=a_{i j}^{T T}+a_{i j}^{T}+\partial_{i} a_{j}+\partial_{j} a_{i}
$$

The components are defined in terms of $a^{T}$,

$$
a^{T}=a-\frac{1}{\nabla^{2}} \partial^{i} \partial^{j} a_{i j}
$$

and are given by,

$$
\begin{aligned}
a_{i} & =\frac{1}{\nabla^{2}}\left(\partial^{j} a_{i j}-\frac{1}{2} \frac{1}{\nabla^{2}} \partial_{i} \partial^{j} \partial^{k} a_{j k}\right), \\
a_{i j}^{T} & =\frac{1}{2}\left(\delta_{i j} a^{T}-\frac{1}{\nabla^{2}} \partial_{i} \partial_{j} a^{T}\right), \\
a_{i j}^{T T} & =a_{i j}-a_{i j}^{T}-\partial_{i} a_{j}-\partial_{j} a_{i} .
\end{aligned}
$$

Here, $a$ is the trace of $a_{i j}$, defined as $a=a_{i j} \delta^{i j}$, and $1 / \nabla^{2}$ is the inverse of the flat space Laplacian. 
In the $\beta_{1}$-model, the linearized equations of motion become

$$
\begin{aligned}
\partial_{t} \hat{\gamma}_{i j}= & \frac{2}{M_{g}^{2}}\left(\pi_{i j}-\frac{1}{2} \delta_{i j} \pi\right)+\partial_{i} L_{j}+\partial_{j} L_{i}+\frac{2}{m^{4} \beta_{1}}\left(\partial_{i} \partial^{k} \pi_{j k}+\partial_{j} \partial^{k} \pi_{i k}\right), \\
\partial_{t} \hat{\phi}_{i j}= & \frac{2}{M_{f}^{2}}\left(p_{i j}-\frac{1}{2} \delta_{i j} p\right)+\partial_{i} L_{j}+\partial_{j} L_{i}, \\
\partial_{t} \pi^{i j}= & -\frac{M_{g}^{2}}{2}\left(\partial_{k} \partial^{i} \hat{\gamma}^{j k}+\partial_{k} \partial^{j} \hat{\gamma}^{i k}-\partial^{i} \partial^{j} \hat{\gamma}-\nabla^{2} \hat{\gamma}^{i j}\right) \\
& +\frac{M_{g}^{2}}{2} \delta^{i j}\left(\partial^{k} \partial^{l} \hat{\gamma}_{k l}-\nabla^{2} \hat{\gamma}\right)+M_{g}^{2}\left(\partial^{i} \partial^{j} \delta N-\delta^{i j} \nabla^{2} \delta N\right) \\
& -m^{4} \beta_{1}\left(\frac{1}{2} \hat{\phi}^{i j}-\frac{1}{2} \hat{\gamma}^{i j}-\delta^{i j} \frac{\hat{\phi}}{2}+\delta^{i j} \frac{\hat{\gamma}}{2}-\delta^{i j} \delta L+\delta^{i j} \delta N\right), \\
\partial_{t} p^{i j}= & -\frac{M_{f}^{2}}{2}\left(\partial_{k} \partial^{i} \hat{\phi}^{j k}+\partial_{k} \partial^{j} \hat{\phi}^{i k}-\partial^{i} \partial^{j} \hat{\phi}-\nabla^{2} \hat{\phi}^{i j}\right) \\
& +\frac{M_{f}^{2}}{2} \delta^{i j}\left(\partial^{k} \partial^{l} \hat{\phi}_{k l}-\nabla^{2} \hat{\phi}\right)+M_{f}^{2}\left(\partial^{i} \partial^{j} \delta L-\delta^{i j} \nabla^{2} \delta L\right) \\
& +m^{4} \beta_{1}\left(\frac{1}{2} \hat{\phi}^{i j}-\frac{1}{2} \hat{\gamma}^{i j}-\delta^{i j} \frac{\hat{\phi}}{2}+\delta^{i j} \frac{\hat{\gamma}}{2}-\delta^{i j} \delta L+\delta^{i j} \delta N\right),
\end{aligned}
$$

where, in the derivation of (A.10), we have used

$$
n^{i}=\frac{\phi^{i j} R_{j}^{(g)}}{\sqrt{4 m^{8} \beta_{1}^{2} \operatorname{det} \gamma+R_{k}^{(g)} \phi^{k l} R_{l}^{(g)}}},
$$

which is the solution to (2.30) in the $\beta_{1}$-model [10]. In addition, the constraints $\tilde{R}^{0}=0$ and $\mathcal{C}=0$, with $\tilde{R}^{0}$ and $\mathcal{C}$ given by (2.20) and (2.22), are linearized to

$$
\begin{aligned}
& M_{f}^{2}\left(\partial^{i} \partial^{j} \hat{\phi}_{i j}-\nabla^{2} \hat{\phi}\right)+m^{4} \beta_{1}(\hat{\gamma}-\hat{\phi})=0 \\
& M_{g}^{2}\left(\partial^{i} \partial^{j} \hat{\gamma}_{i j}-\nabla^{2} \hat{\gamma}\right)+m^{4} \beta_{1}(\hat{\phi}-\hat{\gamma})=0 .
\end{aligned}
$$

In order to determine $L$ and $L^{i}$, coordinate conditions must be imposed. Here we follow a prescription outlined in [3] and impose coordinate conditions with respect to the composite metric,

$$
h_{\mu \nu}=g_{\mu \lambda}\left(\sqrt{g^{-1} f}\right)_{\nu}^{\lambda},
$$

which results in simpler equations. The null cone of $h_{\mu \nu}$ always encloses the intersection of the null cones of $g_{\mu \nu}$ and $f_{\mu \nu}$ [3]. Carrying out a $3+1$ decomposition of $h_{\mu \nu}$ around a flat background,

$$
H \equiv\left(-h^{00}\right)^{-1 / 2}=1+\delta H, \quad H_{i} \equiv h_{0 i}=\delta H_{i}, \quad{ }^{3} h_{i j} \equiv h_{i j}=\delta_{i j}+\hat{h}_{i j},
$$

we obtain, at the linear level,

$$
\hat{h}_{i j}=\frac{1}{2}\left(\hat{\gamma}_{i j}+\hat{\phi}_{i j}\right)
$$


The canonical momentum of ${ }^{3} h_{i j}$ is given by

$$
\pi_{h}^{i j}=2\left(\pi^{i j}+p^{i j}\right) .
$$

We can now use this to choose a coordinate condition to fix a time direction, as well as a spacelike hypersurface. In analogy with [38], we choose

$$
\nabla^{2} \pi_{h}-\partial_{i} \partial_{j} \pi_{h}^{i j}=0
$$

which ensures that the time direction lies inside the null cones of both $g_{\mu \nu}$ and $f_{\mu \nu}$. We must also choose coordinate conditions on the hypersurface. In principle the condition $\partial^{j} \hat{h}_{i j}=0$ could be used, to extend the analogy to [38]. However, the equations turns out to be simpler if the condition

$$
\partial^{j} \hat{\phi}_{i j}=0,
$$

is used instead. The coordinate conditions (A.21) and (A.22) can be rewritten as

$$
\begin{aligned}
\pi_{h}^{T} & =0 \Rightarrow \pi^{T}+p^{T}=0, \\
\hat{\phi}_{i} & =0,
\end{aligned}
$$

using the orthogonal decomposition (A.7) [43]. When rewriting them this way, one makes use of

$$
\frac{1}{\nabla^{2}} 0=0
$$

which holds for the vanishing boundary conditions at infinity [44]. Let us now define,

$$
P_{i j} \equiv p_{i j}-\frac{1}{2} \delta_{i j} p
$$

and use $P_{i j}$ to express (A.11) as

$$
\partial_{t} \hat{\phi}_{i j}=\frac{2}{M_{f}^{2}} P_{i j}+\partial_{i} L_{j}+\partial_{j} L_{i} .
$$

From the definition of the vector component, $a_{i}$, in (A.9), it follows that if $a_{i j}=\partial_{i} L_{j}+\partial_{j} L_{i}$ then $a_{i}=L_{i}$. This leads us to the equation

$$
\partial_{t} \hat{\phi}_{i}=\frac{2}{M_{f}^{2}} P_{i}+L_{i}
$$

where

$$
P_{i}=\frac{1}{\nabla^{2}}\left[\partial^{j} p_{i j}-\frac{1}{4} \partial_{i} p-\frac{1}{2} \partial_{i} \frac{1}{\nabla^{2}} \partial^{j} \partial^{k} p_{j k}\right] .
$$

This, together with (A.24), means (A.28) can be rewritten as

$$
L_{i}=\frac{2}{M_{f}^{2}} \frac{1}{\nabla^{2}}\left(\partial^{j} p_{i j}-\frac{1}{4} \partial_{i} p-\frac{1}{2} \partial_{i} \frac{1}{\nabla^{2}} \partial^{j} \partial^{k} p_{j k}\right) .
$$

The Lagrange multipliers $L^{i}$ are therefore determined in terms of the dynamical variables. Note that this is consistent with the boundary conditions, since both $L_{i}$ and $p^{i j}$ vanish at infinity. 
We now turn our attention to $L$. By imposing the linearized constraint (A.15), the linearized $p^{i j}$ equation of motion, (A.13), can be simplified to

$$
\begin{aligned}
\partial_{t} p^{i j}= & -\frac{M_{f}^{2}}{2}\left(\partial_{k} \partial^{i} \hat{\phi}^{j k}+\partial_{k} \partial^{j} \hat{\phi}^{i k}-\partial^{i} \partial^{j} \hat{\phi}-\nabla^{2} \hat{\phi}^{i j}\right) \\
& +M_{f}^{2}\left(\partial^{i} \partial^{j} \delta L-\delta^{i j} \nabla^{2} \delta L\right)+m^{4} \beta_{1}\left(\delta^{i j} \delta N-\delta^{i j} \delta L-\frac{1}{2} \hat{\gamma}^{i j}+\frac{1}{2} \hat{\phi}^{i j}\right) .
\end{aligned}
$$

From this, we derive the equation of motion for $p^{T}$ by computing the trace of the transverse part of each term on the right hand side, using (A.8). This gives the result

$$
\begin{aligned}
\partial_{t} p^{T}= & -\frac{M_{f}^{2}}{2}\left(\partial^{i} \partial^{j} \hat{\phi}_{i j}-\nabla^{2} \hat{\phi}\right)-2 M_{f}^{2} \nabla^{2} \delta L \\
& +2 m^{4} \beta_{1}(\delta N-\delta L)-\frac{m^{4}}{2} \beta_{1}\left(\hat{\gamma}^{T}-\hat{\phi}^{T}\right) .
\end{aligned}
$$

Similarly, using (A.8) and (A.16), the equation of motion for $\pi^{T}$ becomes

$$
\begin{aligned}
\partial_{t} \pi^{T}= & -\frac{M_{g}^{2}}{2}\left(\partial^{i} \partial^{j} \hat{\gamma}_{i j}-\nabla^{2} \hat{\gamma}\right)-2 M_{g}^{2} \nabla^{2} \delta N \\
& -2 m^{4} \beta_{1}(\delta N-\delta L)+\frac{m^{4}}{2} \beta_{1}\left(\hat{\gamma}^{T}-\hat{\phi}^{T}\right) .
\end{aligned}
$$

Adding these equations yields

$$
\begin{aligned}
\partial_{t}\left(\pi^{T}+p^{T}\right)= & -\frac{M_{g}^{2}}{2}\left(\partial^{i} \partial^{j} \hat{\gamma}_{i j}-\nabla^{2} \hat{\gamma}\right)-\frac{M_{f}^{2}}{2}\left(\partial^{i} \partial^{j} \hat{\phi}_{i j}-\nabla^{2} \hat{\phi}\right) \\
& -2 M_{g}^{2} \nabla^{2} \delta N-2 M_{f}^{2} \nabla^{2} \delta L
\end{aligned}
$$

which, using the coordinate condition (A.23) as well as the constraints (A.15) and (A.16), reduces to

$$
2 M_{g}^{2} \nabla^{2} \delta N+2 M_{f}^{2} \nabla^{2} \delta L=0
$$

which in turn simplifies to

$$
M_{g}^{2} \delta N+M_{f}^{2} \delta L=0 .
$$

In order to solve for $\delta L$ and $\delta N$ in terms of the dynamical variables, the linearized version of equation (A.1) is needed. This equation will now be derived. The linearized version of the secondary constraint, equation (3.35), is

$$
\mathcal{C}_{(2)}=-2 \partial_{i} \partial_{j} \pi^{i j}+\beta_{1} \frac{m^{4}}{M_{g}^{2}} \pi-\beta_{1} \frac{m^{4}}{M_{f}^{2}} p,
$$

and the quadratic Hamiltonian is

$$
\begin{aligned}
H=-\int \mathrm{d}^{3} y & \frac{M_{g}^{2}}{2}\left(-\frac{1}{2} \partial_{k} \hat{\gamma}_{i j} \partial^{k} \hat{\gamma}^{i j}+\partial_{i} \hat{\gamma}_{j k} \partial^{j} \hat{\gamma}^{i k}-\partial_{i} \hat{\gamma}^{i j} \partial_{j} \hat{\gamma}+\frac{1}{2} \partial_{k} \hat{\gamma} \partial^{k} \hat{\gamma}\right) \\
& +\frac{M_{f}^{2}}{2}\left(-\frac{1}{2} \partial_{k} \hat{\phi}_{i j} \partial^{k} \hat{\phi}^{i j}+\partial_{i} \hat{\phi}_{j k} \partial^{j} \hat{\phi}^{i k}-\partial_{i} \hat{\phi}^{i j} \partial_{j} \hat{\phi}+\frac{1}{2} \partial_{k} \hat{\phi} \partial^{k} \hat{\phi}\right) \\
& +M_{g}^{2} \delta N\left(\partial_{i} \partial_{j} \hat{\gamma}^{i j}-\nabla^{2} \hat{\gamma}\right)+M_{f}^{2} \delta L\left(\partial_{i} \partial_{j} \hat{\phi}^{i j}-\nabla^{2} \hat{\phi}\right) \\
& +\frac{m^{4} \beta_{1}}{4}\left(\hat{\gamma}^{i j} \hat{\gamma}_{i j}-\hat{\gamma}^{2}+\hat{\phi}^{i j} \hat{\phi}_{i j}-\hat{\phi}^{2}-2 \hat{\gamma}^{i j} \hat{\phi}_{i j}+2 \hat{\gamma} \hat{\phi}\right) \\
& \left.+m^{4} \beta_{1}(\hat{\phi}(\delta N-\delta L)-\hat{\gamma}(\delta N-\delta L))+\hat{\mathcal{H}}(\pi, p)\right]
\end{aligned}
$$


where $\hat{\mathcal{H}}(\pi, p)$ is a function of $\pi^{i j}$ and $p^{i j}$, the exact form of which will not matter in the calculations to come. Since the linearized $\mathcal{C}_{(2)}$ only depends on the momenta, the linearized version of equation (A.1) can be written

$$
\dot{\mathcal{C}}_{(2)}(x)=\left\{\mathcal{C}_{(2)}(x), H\right\}=-\int \mathrm{d}^{3} z\left(\frac{\delta \mathcal{C}_{(2)}(x)}{\delta \pi^{m n}(z)} \frac{\delta H}{\delta \hat{\gamma}_{m n}(z)}+\frac{\delta \mathcal{C}_{(2)}(x)}{\delta p^{m n}(z)} \frac{\delta H}{\delta \hat{\phi}_{m n}(z)}\right),
$$

where $\mathcal{C}_{(2)}$ and $H$ are given by equation (A.37) and (A.38). From these equations it follows that

$$
\begin{aligned}
\frac{\delta \mathcal{C}_{(2)}(x)}{\delta \pi^{m n}(z)}=- & 2 \frac{\partial}{\partial x^{m}} \frac{\partial}{\partial x^{n}} \delta^{3}(x-z)+\beta_{1} \frac{m^{4}}{M_{g}^{2}} \delta_{m n} \delta^{3}(x-z) \\
\frac{\delta \mathcal{C}_{(2)}(x)}{\delta p^{m n}(z)}=- & \beta_{1} \frac{m^{4}}{M_{f}^{2}} \delta_{m n} \delta^{3}(x-z), \\
\frac{\delta H}{\delta \hat{\gamma}(z)}=- & {\left[\frac{M_{g}^{2}}{2}\left(\nabla^{2} \hat{\gamma}^{m n}-\partial_{i} \partial^{m} \hat{\gamma}^{i n}-\partial_{i} \partial^{n} \hat{\gamma}^{i m}+\partial^{m} \partial^{n} \hat{\gamma}+\delta^{m n} \partial_{i} \partial_{j} \hat{\gamma}^{i j}-\delta^{m n} \nabla^{2} \hat{\gamma}\right)\right.} \\
& +M_{g}^{2}\left(\partial^{m} \partial^{n} \delta N-\delta^{m n} \nabla^{2} \delta N\right) \\
& \left.+m^{4} \beta_{1}\left(\frac{1}{2}\left(\hat{\gamma}^{m n}-\delta^{m n} \hat{\gamma}-\hat{\phi}^{m n}+\delta^{m n} \hat{\phi}\right)-\delta^{m n}(\delta N-\delta L)\right)\right] \\
\frac{\delta H}{\delta \hat{\phi}(z)}= & {\left[\frac{M_{f}^{2}}{2}\left(\nabla^{2} \hat{\phi}^{m n}-\partial_{i} \partial^{m} \hat{\phi}^{i n}-\partial_{i} \partial^{n} \hat{\phi}^{i m}+\partial^{m} \partial^{n} \hat{\phi}+\delta^{m n} \partial_{i} \partial_{j} \hat{\phi}^{i j}-\delta^{m n} \nabla^{2} \hat{\phi}\right)\right.} \\
& +M_{f}^{2}\left(\partial^{m} \partial^{n} \delta L-\delta^{m n} \nabla^{2} \delta L\right) \\
& \left.+m^{4} \beta_{1}\left(\frac{1}{2}\left(\hat{\phi}^{m n}-\delta^{m n} \hat{\phi}-\hat{\gamma}^{m n}+\delta^{m n} \hat{\gamma}\right)+\delta^{m n}(\delta N-\delta L)\right)\right] .
\end{aligned}
$$

Inserting these into equation (A.39) yields the result

$$
\begin{aligned}
\dot{\mathcal{C}}_{(2)}=m^{4} \beta_{1} & {\left[\frac{1}{2}\left(\partial_{i} \partial_{j} \hat{\phi}^{i j}-\nabla^{2} \hat{\phi}\right)-\frac{1}{2}\left(\partial_{i} \partial_{j} \hat{\gamma}^{i j}-\nabla^{2} \hat{\gamma}\right)\right.} \\
& \left.+m^{4} \beta_{1}(\hat{\phi}-\hat{\gamma}-3(\delta N-\delta L))\left(\frac{1}{M_{g}^{2}}+\frac{1}{M_{f}^{2}}\right)\right],
\end{aligned}
$$

which, after imposing the constraints (A.15) and (A.16), simplifies to

$$
\dot{\mathcal{C}}_{(2)} \approx 3 m^{8} \beta_{1}^{2}\left(\frac{1}{M_{g}^{2}}+\frac{1}{M_{f}^{2}}\right)\left(\frac{1}{2}(\hat{\phi}-\hat{\gamma})+\delta L-\delta N\right) .
$$

$\dot{\mathcal{C}}_{(2)} \approx 0$ then implies

$$
\delta N-\delta L=\frac{1}{2}(\hat{\phi}-\hat{\gamma})
$$


which, together with equation (A.36), can be solved for $\delta L$ and $\delta N$. The result is

$$
\begin{aligned}
& \delta L=-\frac{M_{g}^{2}}{2\left(M_{g}^{2}+M_{f}^{2}\right)}(\hat{\phi}-\hat{\gamma}), \\
& \delta N=\frac{M_{f}^{2}}{2\left(M_{g}^{2}+M_{f}^{2}\right)}(\hat{\phi}-\hat{\gamma}) .
\end{aligned}
$$

This shows that all Lagrange multipliers in bimetric gravity can be determined, using the consistency condition that $\mathcal{C}_{(2)}$ is preserved in time, as well as suitable coordinate conditions. This procedure can be generalized to the non-linear case, in a manner similar to that described in [38].

\section{B Computation of the Poisson brackets}

Here we compute the Poisson brackets of the constraints $\tilde{R}^{0}, \tilde{R}_{i}, \mathcal{C}$, and $\mathcal{C}_{(2)}$. A computation of the algebra of constraints of general relativity (4.1) can be found in the appendix of [45] to which we refer for the relevant details. In particular, we reproduce the following results that will be useful in the computation of brackets in bimetric theory. To systematically deal with the derivatives of the delta function that arise in the calculations one can introduce smoothing functions and define,

$$
\begin{array}{rlrl}
A_{H}^{(g)} & \equiv \int \mathrm{d}^{3} x a^{0}(x) R^{0(g)}(x), & A^{(g)} \equiv \int \mathrm{d}^{3} x a^{i}(x) R_{i}^{(g)}(x), \\
B_{H}^{(g)} & \equiv \int \mathrm{d}^{3} y b^{0}(y) R^{0(g)}(y), & B^{(g)} & \equiv \int \mathrm{d}^{3} y b^{i}(y) R_{i}^{(g)}(y),
\end{array}
$$

where $a^{0}, a^{i}, b^{0}$ and $b^{i}$ are time independent smoothing functions. These can be used to compute the brackets of the constraints involving the metric $g_{\mu \nu}$, since, for example,

$$
\left\{A^{(g)}, B^{(g)}\right\}=\int \mathrm{d}^{3} x \int \mathrm{d}^{3} y a^{i}(x) b^{i}(y)\left\{R_{i}^{(g)}(x), R_{i}^{(g)}(y)\right\} .
$$

Then, one can obtain the following intermediate relations,

$$
\begin{aligned}
\frac{\delta A^{(g)}}{\delta \gamma_{m n}} & =\sqrt{\operatorname{det} \gamma} \nabla_{i}\left(a^{i} \frac{\pi^{m n}}{\sqrt{\operatorname{det} \gamma}}\right)-\pi^{m i} \nabla_{i} a^{n}-\pi^{n i} \nabla_{i} a^{m}, \\
\frac{\delta A^{(g)}}{\delta \pi^{m n}} & =-\left(\gamma_{n j} \nabla_{m} a^{j}+\gamma_{m j} \nabla_{n} a^{j}\right), \\
\frac{\delta A_{H}^{(g)}}{\delta \pi^{m n}} & =-\frac{a^{0}}{M_{g}^{2} \sqrt{\operatorname{det} \gamma}}\left(2 \pi_{m n}-\gamma_{m n} \pi^{k} k\right), \\
\left\{A^{(g)}, B^{(g)}\right\} & =-\int \mathrm{d}^{3} z\left(a^{i} R_{j}^{(g)} \nabla_{i} b^{j}-b^{j} R_{i}^{(g)} \nabla_{j} a^{i}\right), \\
\left\{A_{H}^{(g)}, B^{(g)}\right\} & =-\int \mathrm{d}^{3} z a^{0} \nabla_{i}\left(b^{i} R^{0(g)}\right), \\
\left\{A_{H}^{(g)}, B_{H}^{(g)}\right\} & =-\int \mathrm{d}^{3} z\left(a^{0} R^{i(g)} \nabla_{i} b^{0}-b^{0} R^{i(g)} \nabla_{i} a^{0}\right) .
\end{aligned}
$$


Similarly, in analogy with (B.1) and (B.2), for the $f_{\mu \nu}$ metric one can define,

$$
\begin{array}{rlrl}
A_{H}^{(f)} & \equiv \int \mathrm{d}^{3} x a^{0}(x) R^{0(f)}(x), & A^{(f)} \equiv \int \mathrm{d}^{3} x a^{i}(x) R_{i}^{(f)}(x), \\
B_{H}^{(f)} & \equiv \int \mathrm{d}^{3} y b^{0}(y) R^{0(f)}(y), & B^{(f)} & \equiv \int \mathrm{d}^{3} y b^{i}(y) R_{i}^{(f)}(y),
\end{array}
$$

which satisfy the same relations as those for the $g_{\mu \nu}$ metric above.

\section{B.1 Evaluation of $\left\{\tilde{\boldsymbol{R}}^{0}(x), \tilde{\boldsymbol{R}}_{i}(y)\right\}$}

Corresponding to the bimetric constraints $\tilde{R}^{0}(2.20)$ and $\tilde{R}_{i}(2.21)$, let us introduce the smeared functions,

$$
F \equiv \int \mathrm{d}^{3} x f(x) \tilde{R}^{0}(x), \quad G \equiv \int \mathrm{d}^{3} y g^{i}(y) \tilde{R}_{i}(y),
$$

where, $f(x)$ and $g^{i}(y)$ are time independent smoothing functions. It follows that

$$
\{F, G\}=\int \mathrm{d}^{3} x \int \mathrm{d}^{3} y f(x) g^{i}(y)\left\{\tilde{R}^{0}(x), \tilde{R}_{i}(y)\right\} .
$$

From the definition of the Poisson bracket, this can also be written as

$$
\begin{aligned}
\{F, G\}=\{F, G\}_{g}+\{F, G\}_{f}= & \int \mathrm{d}^{3} z\left(\frac{\delta F}{\delta \gamma_{m n}(z)} \frac{\delta G}{\delta \pi^{m n}(z)}-\frac{\delta F}{\delta \pi^{m n}(z)} \frac{\delta G}{\delta \gamma_{m n}(z)}\right) \\
& +\int \mathrm{d}^{3} z\left(\frac{\delta F}{\delta \phi_{m n}(z)} \frac{\delta G}{\delta p^{m n}(z)}-\frac{\delta F}{\delta p^{m n}(z)} \frac{\delta G}{\delta \phi_{m n}(z)}\right)
\end{aligned}
$$

The variations of $G$ with respect to $\gamma_{i j}$ and $\pi^{i j}$ are given by (B.4) and (B.5),

$$
\begin{aligned}
\frac{\delta G}{\delta \gamma_{m n}} & =\sqrt{\operatorname{det} \gamma} \nabla_{i}\left(g^{i} \frac{\pi^{m n}}{\sqrt{\operatorname{det} \gamma}}\right)-\pi^{m i} \nabla_{i} g^{n}-\pi^{n i} \nabla_{i} g^{m}, \\
\frac{\delta G}{\delta \pi^{m n}} & =-\left(\gamma_{n j} \nabla_{m} g^{j}+\gamma_{m j} \nabla_{n} g^{j}\right) .
\end{aligned}
$$

The variations with respect to $\phi_{i j}$ and $p^{i j}$ are given by the analogue of (B.4) and (B.5) for $f_{\mu \nu}$,

$$
\begin{aligned}
\frac{\delta G}{\delta \phi_{m n}} & =\sqrt{\operatorname{det} \phi} \bar{\nabla}_{i}\left(g^{i} \frac{p^{m n}}{\sqrt{\operatorname{det} \phi}}\right)-p^{m i} \bar{\nabla}_{i} g^{n}-p^{n i} \bar{\nabla}_{i} g^{m}, \\
\frac{\delta G}{\delta p^{m n}} & =-\left(\phi_{n j} \bar{\nabla}_{m} g^{j}+\phi_{m j} \bar{\nabla}_{n} g^{j}\right) .
\end{aligned}
$$

From equation (2.27), it follows that when computing the variations of $F$, we need not consider the implicit dependence on the dynamical variables through $n^{i}$, but can in fact keep $n^{i}$ fixed. It is therefore useful to introduce the notation,

$$
f^{i}=f n^{i}
$$


and define the quantities,

$$
B_{F} \equiv \int \mathrm{d}^{3} x f(x) R^{0(f)}(x), \quad D_{F} \equiv \int \mathrm{d}^{3} x f^{i}(x) R_{i}^{(g)}(x) .
$$

From these definitions, and (2.20), it follows that,

$$
\begin{aligned}
\frac{\delta F}{\delta \gamma_{m n}} & =\frac{\delta D_{F}}{\delta \gamma_{m n}}+2 m^{4} f \frac{\partial\left(\sqrt{\operatorname{det} \gamma} U^{\prime}\right)}{\partial \gamma_{m n}}, & \frac{\delta F}{\delta \pi^{m n}} & =\frac{\delta D_{F}}{\delta \pi^{m n}}, \\
\frac{\delta F}{\delta \phi_{m n}} & =\frac{\delta B_{F}}{\delta \phi_{m n}}+2 m^{4} f \sqrt{\operatorname{det} \gamma} \frac{\partial U^{\prime}}{\partial \phi_{m n}}, & \frac{\delta F}{\delta p^{m n}} & =\frac{\delta B_{F}}{\delta p^{m n}} .
\end{aligned}
$$

Then the bracket $\{F, G\}_{g}$ can then be written as,

$$
\{F, G\}_{g}=\left\{D_{F}, G\right\}_{g}+2 m^{4} \int \mathrm{d}^{3} z f \frac{\partial\left(\sqrt{\operatorname{det} \gamma} U^{\prime}\right)}{\partial \gamma_{m n}} \frac{\delta G}{\delta \pi^{m n}} .
$$

Since $n^{i}$ is kept fixed, the first term is similar in form to (B.7), and is therefore given by

$$
\left\{D_{F}, G\right\}_{g}=-\int \mathrm{d}^{3} z\left(f^{i} R_{j}^{(g)} \nabla_{i} g^{j}-g^{j} R_{i}^{(g)} \nabla_{j} f^{i}\right) .
$$

The second term is ( since $\sqrt{\operatorname{det} \gamma} U^{\prime}$ and $\sqrt{\operatorname{det} \gamma} U$ differ by a term independent of $\gamma$ ),

$$
2 m^{4} \int \mathrm{d}^{3} z f \frac{\partial\left(\sqrt{\operatorname{det} \gamma} U^{\prime}\right)}{\partial \gamma_{m n}} \frac{\delta G}{\delta \pi^{m n}}=-2 m^{4} \int \mathrm{d}^{3} z f \sqrt{\operatorname{det} \gamma}\left(U \nabla_{j} g^{j}+2 \frac{\partial U}{\partial \gamma_{m n}} \gamma_{n j} \nabla_{m} g^{j}\right) .
$$

Now we turn our attention to $\{F, G\}_{f}$, which can be written as,

$$
\{F, G\}_{f}=\left\{B_{F}, G\right\}_{f}+2 m^{4} \int \mathrm{d}^{3} z f \sqrt{\operatorname{det} \gamma} \frac{\partial U^{\prime}}{\partial \phi_{m n}} \frac{\delta G}{\delta p^{m n}} .
$$

The first term is analogous to (B.8), and is therefore given by

$$
\left\{B_{F}, G\right\}_{f}=-\int \mathrm{d}^{3} z f \partial_{i}\left(g^{i} R^{0(f)}\right) .
$$

Here as have used

$$
\bar{\nabla}_{i}\left(g^{i} R^{0(f)}\right)=\partial_{i}\left(g^{i} R^{0(f)}\right)
$$

which follows since $g^{i}$ is a vector and $R^{0(f)}$ is a scalar density. ${ }^{9}$ The second term becomes,

$$
2 m^{4} \int \mathrm{d}^{3} z f \sqrt{\operatorname{det} \gamma} \frac{\partial U^{\prime}}{\partial \phi_{m n}} \frac{\delta G}{\delta p^{m n}}=-2 m^{4} \int \mathrm{d}^{3} z f \sqrt{\operatorname{det} \gamma} \tilde{U}^{m n} \phi_{n j} \bar{\nabla}_{m} g^{j},
$$

where $\tilde{U}^{m n}$ is defined by (A.5). Putting the above results together, one obtains,

$$
\begin{aligned}
\{F, G\}= & \int \mathrm{d}^{3} z\left[-f \partial_{i}\left(g^{i} R^{0(f)}\right)-f^{i} R_{j}^{(g)} \nabla_{i} g^{j}+g^{j} R_{i}^{(g)} \nabla_{j} f^{i}\right. \\
& \left.-2 m^{4} f \sqrt{\operatorname{det} \gamma}\left(U \nabla_{i} g^{i}+2 \frac{\partial U}{\partial \gamma_{m n}} \gamma_{n j} \nabla_{m} g^{j}+\tilde{U}^{m n} \phi_{n j} \bar{\nabla}_{m} g^{j}\right)\right] .
\end{aligned}
$$

\footnotetext{
${ }^{9}$ To derive the identity, one needs the formula for the covariant derivative of a scalar density, $\nabla_{\alpha} D=$ $\partial_{\alpha} D-\Gamma_{\alpha \mu}^{\mu} D$.
} 
We now bring (B.30) to the appropriate form by rewriting the terms. Using integration by parts, the third term can be rewritten as,

$$
\int \mathrm{d}^{3} z g^{j} R_{i}^{(g)} \nabla_{j} f^{i}=-\int \mathrm{d}^{3} z f \partial_{i}\left(g^{i} n^{j} R_{j}^{(g)}\right)+\int \mathrm{d}^{3} z f g^{i} R_{j}^{(g)} \nabla_{i} n^{j} .
$$

Using (2.23) and (A.5), it is possible to write,

$$
f \sqrt{\operatorname{det} \gamma} \tilde{U}^{m n} \phi_{n j} \bar{\nabla}_{m} g^{j}=2 f \sqrt{\operatorname{det} \gamma} \frac{\partial U}{\partial \phi_{m n}} \phi_{n j} \bar{\nabla}_{m} g^{j}+\beta_{4} f \sqrt{\operatorname{det} \phi} \bar{\nabla}_{i} g^{i} .
$$

The last term in the above expression, together with the fourth term in (B.30), can be rewritten in the following manner,

$$
\int \mathrm{d}^{3} z f\left(\sqrt{\operatorname{det} \gamma} U \nabla_{i} g^{i}+\beta_{4} \sqrt{\operatorname{det} \phi} \bar{\nabla}_{i} g^{i}\right)=\int \mathrm{d}^{3} z f\left(\partial_{i}\left(g^{i} \sqrt{\operatorname{det} \gamma} U^{\prime}\right)-g^{i} \sqrt{\operatorname{det} \gamma} \partial_{i} U\right) .
$$

Putting these results into (B.30), and using (2.20), we get,

$$
\begin{aligned}
\{F, G\}= & \int \mathrm{d}^{3} z\left[-f \partial_{i}\left(g^{i} \tilde{R}^{0}\right)+f g^{i} R_{j}^{(g)} \nabla_{i} n^{j}-f^{i} R_{j}^{(g)} \nabla_{i} g^{j}\right. \\
& \left.+2 m^{4} f \sqrt{\operatorname{det} \gamma}\left(g^{i} \partial_{i} U-2 \frac{\partial U}{\partial \gamma_{m n}} \gamma_{n j} \nabla_{m} g^{j}-2 \frac{\partial U}{\partial \phi_{m n}} \phi_{n j} \bar{\nabla}_{m} g^{j}\right)\right] .
\end{aligned}
$$

The first term in this expression is the one that appears in the covariance algebra. Thus, to obtain the correct algebra, all other terms must cancel. Note that, since $f^{i}=f n^{i}$, we can write,

$$
f g^{i} R_{j}^{(g)} \nabla_{i} n^{j}-f^{i} R_{j}^{(g)} \nabla_{i} g^{j}=f g^{i} R_{j}^{(g)} \partial_{i} n^{j}-f^{i} R_{j}^{(g)} \partial_{i} g^{j}
$$

In the second line, writing $U=\beta_{1}\left[U_{1}\right]+\beta_{2}\left[U_{2}\right]+\beta_{3}\left[U_{3}\right]$, the derivative $\frac{\partial U}{\partial \gamma_{m n}}$ was computed in equations (3.29)-(3.31). Now we evaluate $\frac{\partial U}{\partial \phi_{m n}}$ in a similar way. The $\beta_{1}$-term gives,

$$
\beta_{1} \frac{\partial\left[U_{1}\right]}{\partial \phi_{m n}}=\beta_{1} \frac{\partial \sqrt{x}}{\partial \phi_{m n}}=-\frac{\beta_{1}}{2} \frac{n^{m} n^{n}}{\sqrt{x}} .
$$

The $\beta_{2}$-term, on using equation (3.6), becomes

$$
\beta_{2} \frac{\partial\left[U_{2}\right]}{\partial \phi_{m n}}=\beta_{2}\left(-\frac{n^{m} n^{n}}{2} D^{i}{ }_{i}+n^{(m} D_{k}^{n)} n^{k}+\frac{1}{2} Q_{1}^{m n}\right) .
$$

The $\beta_{3}$-term, on using (3.6) and (3.7), becomes,

$$
\begin{aligned}
\beta_{3} \frac{\partial\left[U_{3}\right]}{\partial \phi_{m n}}= & \beta_{3} \sqrt{x}\left[\frac{1}{\sqrt{x}} \frac{\partial\left(\sqrt{x} D_{l}^{l}\right)}{\partial \phi_{m n}} n^{i} \phi_{i j} D^{j}{ }_{k} n^{k}-D^{i}{ }_{k} n^{k} \phi_{i j} \frac{\partial\left(D^{j}{ }_{l} n^{l}\right)}{\partial \phi_{m n}}+\frac{n^{m} n^{n}}{2 x} D^{i}{ }_{k} n^{k} \phi_{i j} D^{j}{ }_{l} n^{l}\right. \\
& \left.+D^{l}{ }_{l} n^{(m} D^{n)}{ }_{k} n^{k}-D^{m}{ }_{k} n^{k} D^{n}{ }_{l} n^{l}-\frac{n^{m} n^{n}}{2} e_{2}(D)+\frac{1}{2} D^{l}{ }_{l} Q_{1}^{m n}-\frac{1}{2} Q_{2}^{m n}\right] .
\end{aligned}
$$


Using (3.6), the first two terms of this can be rewritten as,

$$
\begin{aligned}
& \frac{\partial\left(\sqrt{x} D^{l}{ }_{l}\right)}{\partial \phi_{m n}} n^{i} \phi_{i j} D^{j}{ }_{k} n^{k}-\sqrt{x} D^{i}{ }_{k} n^{k} \phi_{i j} \frac{\partial\left(D^{j}{ }_{l} n^{l}\right)}{\partial \phi_{m n}} \\
& \quad=-n^{l} \phi_{l i}\left(\sqrt{x} D^{i}{ }_{j}+\frac{1}{\sqrt{x}} D^{i}{ }_{r} n^{r} n^{q} \phi_{q j}\right) \frac{\partial\left(D^{j}{ }_{k} n^{k}\right)}{\partial \phi_{m n}}+\frac{1}{2} \frac{1}{\sqrt{x}} n^{i} \phi_{i j} D^{j}{ }_{k} n^{k} Q_{1}^{m n} .
\end{aligned}
$$

When combined with the relation ${ }^{10}$

$$
\begin{aligned}
n^{l} \phi_{l i} & \left(\sqrt{x} D^{i}{ }_{j}+\frac{1}{\sqrt{x}} D^{i}{ }_{r} n^{r} n^{q} \phi_{q j}\right) \frac{\partial\left(D^{j}{ }_{k} n^{k}\right)}{\partial \phi_{m n}} \\
& =\frac{1}{2} \frac{n^{m} n^{n}}{\sqrt{x}} D^{i}{ }_{k} n^{k} \phi_{i j} D^{j}{ }_{l} n^{l}+\sqrt{x} n^{(m} D^{n)}{ }_{l} D^{l}{ }_{k} n^{k}-\frac{1}{2} \sqrt{x} D^{m}{ }_{k} n^{k} D^{n}{ }_{l} n^{l},
\end{aligned}
$$

this implies that the $\beta_{3}$-term becomes,

$$
\begin{aligned}
\beta_{3} \frac{\partial\left[U_{3}\right]}{\partial \phi_{m n}}= & \beta_{3} \sqrt{x}\left[D^{l}{ }_{l} n^{(m} D^{n)}{ }_{k} n^{k}-n^{(m} D^{n)}{ }_{l} D^{l}{ }_{k} n^{k}-\frac{1}{2} D^{m}{ }_{k} n^{k} D^{n}{ }_{l} n^{l}\right. \\
& \left.-\frac{n^{m} n^{n}}{4}\left(D^{i}{ }_{i} D^{j}{ }_{j}-D^{i}{ }_{j} D^{j}{ }_{i}\right)+\frac{1}{2} D^{l}{ }_{l} Q_{1}^{m n}-\frac{1}{2} Q_{2}^{m n}+\frac{1}{2 x} n^{i} \phi_{i j} D^{j}{ }_{k} n^{k} Q_{1}^{m n}\right] .
\end{aligned}
$$

Putting these together gives $\partial U / \partial \phi_{m n}$.

Now, observe that the last two terms of (B.34) can be written as,

$$
\begin{aligned}
& 2 \frac{\partial U}{\partial \gamma_{m n}} \gamma_{n j} \nabla_{m} g^{j}+2 \frac{\partial U}{\partial \phi_{m n}} \phi_{n j} \bar{\nabla}_{m} g^{j} \\
& \quad=\left(2 \frac{\partial U}{\partial \gamma_{m n}} \gamma_{n j}+2 \frac{\partial U}{\partial \phi_{m n}} \phi_{n j}\right) \partial_{m} g^{j}+2 \frac{\partial U}{\partial \gamma_{m n}} \gamma_{n j} \Gamma_{m i}^{j} g^{i}+2 \frac{\partial U}{\partial \phi_{m n}} \phi_{n j} \bar{\Gamma}_{m i}^{j} g^{i} .
\end{aligned}
$$

The quantity in the parenthesis is computed using (3.29)-(3.31) and (B.36)-(B.41). Its $\beta_{1}$-term is,

$$
\left(2 \frac{\partial U}{\partial \gamma_{m n}} \gamma_{n j}+2 \frac{\partial U}{\partial \phi_{m n}} \phi_{n j}\right)_{\beta_{1}}=-\beta_{1} n^{m} \frac{n^{l} \phi_{l i}}{\sqrt{x}} \delta^{i}{ }_{j}
$$

After a short calculation, using (3.9), the $\beta_{2}$-terms become,

$$
\left(2 \frac{\partial U}{\partial \gamma_{m n}} \gamma_{n j}+2 \frac{\partial U}{\partial \phi_{m n}} \phi_{n j}\right)_{\beta_{2}}=-\beta_{2} n^{m} \frac{n^{l} \phi_{l i}}{\sqrt{x}} \sqrt{x}\left(\delta^{i}{ }_{j} D_{k}^{k}-D_{j}^{i}\right) .
$$

Finally, the $\beta_{3}$-term can be written as,

$$
\begin{aligned}
& \left(2 \frac{\partial U}{\partial \gamma_{m n}} \gamma_{n j}+2 \frac{\partial U}{\partial \phi_{m n}} \phi_{n j}\right)_{\beta_{3}} \\
& \quad=-\beta_{3} n^{m} \frac{n^{l} \phi_{l i}}{\sqrt{x}} x\left(\frac{1}{2} \delta^{i}{ }_{j}\left(D_{r}^{r} D_{k}^{k}-D_{k}^{r} D_{r}^{k}\right)+D_{k}^{i} D_{j}^{k}-D_{j}^{i} D_{k}^{k}\right)+\beta_{3} A_{j}^{m}
\end{aligned}
$$

\footnotetext{
${ }^{10}$ Equation (B.40) is analogous to equation (4.6) in [10], and is derived in a similar way.
} 
where $A_{j}^{m}$ are given by,

$$
\begin{aligned}
A_{j}^{m}= & \sqrt{x}\left[D_{l}^{l}\left(n^{n} D^{m}{ }_{k} n^{k}+Q_{1}^{m n}\right)-\left(n^{n} D^{m}{ }_{l}-\frac{1}{x} n^{i} \phi_{i l} Q_{1}^{m n}\right) D_{k}^{l} n^{k}-D^{m}{ }_{k} n^{k} D^{n}{ }_{l} n^{l}-Q_{2}^{m n}\right] \phi_{n j} \\
& +\sqrt{x}\left[D^{l}{ }_{l} \phi_{i r}\left(D^{-1}\right)^{r}{ }_{k}-\phi_{k i}+\frac{1}{x} n^{l} \phi_{l q} D^{q}{ }_{r} n^{r} \phi_{i s}\left(D^{-1}\right)^{s}{ }_{k}-\frac{1}{x} n^{r} \phi_{r i} n^{l} \phi_{l k}\right] \frac{\partial \gamma^{k i}}{\partial \gamma_{m n}} \gamma_{n j} .
\end{aligned}
$$

Using (3.9) and (3.10), this reduces to,

$$
A_{j}^{m}=-\left(\frac{1}{\sqrt{x}} n^{s} \phi_{s q} D^{q}{ }_{r} n^{r} D^{m}{ }_{i} n^{i}+\sqrt{x} D^{m}{ }_{l} D_{k}^{l} n^{k}\right) n^{n} \phi_{n j}-\frac{1}{\sqrt{x}} n^{i} \phi_{i r} n^{l} \phi_{l k} \frac{\partial \gamma^{r k}}{\partial \gamma_{m n}} \gamma_{n j}=0 .
$$

The vanishing of $A_{j}^{m}$ follows from the definition of the matrix $D$ in (2.16), which implies,

$$
x D^{m}{ }_{l} D^{l}{ }_{k}=\gamma^{m l} \phi_{l k}-D^{m}{ }_{i} n^{i} D^{l}{ }_{j} n^{j} \phi_{l k},
$$

Putting (B.43), (B.44) and (B.45) together, we get,

$$
\begin{aligned}
2 m^{4} f & \sqrt{\operatorname{det} \gamma}\left(-2 \frac{\partial U}{\partial \gamma_{m n}} \gamma_{n j}-2 \frac{\partial U}{\partial \phi_{m n}} \phi_{n j}\right) \partial_{m} g^{j} \\
= & 2 m^{4} f^{m} \sqrt{\operatorname{det} \gamma} \frac{n^{l} \phi_{l i}}{\sqrt{x}}\left[\beta_{1} \delta^{i}{ }_{j}+\beta_{2} \sqrt{x}\left(\delta^{i}{ }_{j} D_{k}^{k}-D^{i}{ }_{j}\right)\right. \\
& \left.+\beta_{3} x\left(\frac{1}{2} \delta^{i}{ }_{j}\left(D^{r}{ }_{r} D^{k}{ }_{k}-D^{r}{ }_{k} D^{k}{ }_{r}\right)+D^{i}{ }_{k} D^{k}{ }_{j}-D^{i}{ }_{j} D^{k}{ }_{k}\right)\right] \partial_{m} g^{j}=f^{i} R_{j}^{(g)} \partial_{i} g^{j},
\end{aligned}
$$

where (2.28) and (2.30) have been used in the last step. This means that (B.34) simplifies to

$$
\begin{aligned}
\{F, G\}=\int \mathrm{d}^{3} z\left[-f \partial_{i}\left(g^{i} \tilde{R}^{0}\right)+f g^{i} R_{j}^{(g)} \partial_{i} n^{j}\right. \\
\left.\quad+2 m^{4} f \sqrt{\operatorname{det} \gamma}\left(g^{i} \partial_{i} U-2 \frac{\partial U}{\partial \gamma_{m n}} \gamma_{n j} \Gamma_{m i}^{j} g^{i}-2 \frac{\partial U}{\partial \phi_{m n}} \phi_{n j} \bar{\Gamma}_{m i}^{j} g^{i}\right)\right] .
\end{aligned}
$$

The final two terms of this can be rewritten as,

$$
2 \frac{\partial U}{\partial \gamma_{m n}} \gamma_{n j} \Gamma_{m i}^{j} g^{i}=g^{i} \frac{\partial U}{\partial \gamma_{m n}} \partial_{i} \gamma_{m n}, \quad 2 \frac{\partial U}{\partial \phi_{m n}} \phi_{n j} \bar{\Gamma}_{m i}^{j} g^{i}=g^{i} \frac{\partial U}{\partial \phi_{m n}} \partial_{i} \phi_{m n} .
$$

Furthermore, the derivative of $U$ can be expressed as,

$$
\partial_{i} U=\frac{\partial U}{\partial n^{j}} \partial_{i} n^{j}+\frac{\partial U}{\partial \gamma_{m n}} \partial_{i} \gamma_{m n}+\frac{\partial U}{\partial \phi_{m n}} \partial_{i} \phi_{m n} .
$$

Note that the last two terms in this expression cancels against the terms involving Christoffel symbols in (B.50). Finally, from (2.27), it follows that,

$$
2 m^{4} \sqrt{\operatorname{det} \gamma} f g^{i} \frac{\partial U}{\partial n^{j}} \partial_{i} n^{j}=-f g^{i} R_{j}^{(g)} \partial_{i} n^{j},
$$

which cancels against the second term in (B.50). The bracket (B.50) therefore becomes

$$
\{F, G\}=-\int \mathrm{d}^{3} z f \partial_{i}\left(g^{i} \tilde{R}^{0}\right) .
$$

In view of (B.13), this yields bracket quoted in (4.4),

$$
\left\{\tilde{R}^{0}(x), \tilde{R}_{i}(y)\right\}=-\tilde{R}^{0}(y) \frac{\partial}{\partial x^{i}} \delta^{3}(x-y) .
$$




\section{B.2 Evaluation of $\left\{\tilde{R}^{0}(x), \tilde{R}^{0}(y)\right\}$}

In analogy with $F, B_{F}$ and $D_{F}$ defined as in (B.12) and (B.20), let us also define,

$$
K \equiv \int \mathrm{d}^{3} y k(y) \tilde{R}^{0}(y), \quad B_{K} \equiv \int \mathrm{d}^{3} y k(y) R^{0(f)}(y), \quad D_{K} \equiv \int \mathrm{d}^{3} y k^{i}(y) R_{i}^{(g)}(y),
$$

where, $k^{i}=k n^{i}$ and, as in the previous section, $n^{i}$ can be kept fixed when computing the variations. From this, it follows that,

$$
\{F, K\}=\int \mathrm{d}^{3} x \int \mathrm{d}^{3} y f(x) k(y)\left\{\tilde{R}^{0}(x), \tilde{R}^{0}(y)\right\} .
$$

The variations of $F$ are once again given by (B.21), and (B.22), while the variations of $K$ are given by similar equations with smoothing function $f$ replaced by $k$. As before, the bracket $\{F, K\}$ can be split in two parts, $\{F, K\}=\{F, K\}_{g}+\{F, K\}_{f}$, the first of these is given by,

$$
\{F, K\}_{g}=\left\{D_{F}, D_{K}\right\}_{g}+2 m^{4} \int \mathrm{d}^{3} z \frac{\partial\left(\sqrt{\operatorname{det} \gamma} U^{\prime}\right)}{\partial \gamma_{m n}}\left(f \frac{\delta D_{K}}{\delta \pi^{m n}}-k \frac{\delta D_{F}}{\delta \pi^{m n}}\right) .
$$

The variations $\delta D_{F} / \delta \pi^{m n}$ and $\delta D_{K} / \delta \pi^{m n}$ are given by (B.5), and the bracket $\left\{D_{F}, D_{K}\right\}_{g}$ is similar to (B.7). Putting all this together gives,

$$
\{F, K\}_{g}=\int \mathrm{d}^{3} z\left[2 m^{4} \sqrt{\operatorname{det} \gamma} U^{m n} \gamma_{n j}\left(k^{j} \partial_{m} f-f^{j} \partial_{m} k\right)-\left(f^{i} R_{j}^{(g)} \partial_{i} k^{j}-k^{j} R_{i}^{(g)} \partial_{j} f^{i}\right)\right],
$$

where the covariant derivatives have been replaced with ordinary ones since the terms involving Christoffel symbols cancel.

Now we turn our attention to $\{F, K\}_{f}$. This bracket is given by,

$$
\{F, K\}_{f}=\left\{B_{F}, B_{K}\right\}_{f}+2 m^{4} \int \mathrm{d}^{3} z \sqrt{\operatorname{det} \gamma} \frac{\partial U^{\prime}}{\partial \phi_{m n}}\left(f \frac{\delta B_{K}}{\delta p^{m n}}-k \frac{\delta B_{F}}{\delta p^{m n}}\right) .
$$

The variations of $B_{F}$ and $B_{K}$ with respect to $p^{i j}$ are given by (B.6) and the terms under the integral sign cancel out. The bracket $\left\{B_{F}, B_{K}\right\}$ is given by (B.9), now with $R^{i(f)} \equiv \phi^{i j} R_{j}^{(f)}$. Hence,

$$
\{F, K\}_{f}=\left\{B_{F}, B_{K}\right\}_{f}=-\int \mathrm{d}^{3} z\left(f R^{i(f)} \partial_{i} k-k R^{i(f)} \partial_{i} f\right) .
$$

Combining $\{F, K\}_{f}$ and $\{F, K\}_{g}$ yields the expression

$$
\begin{aligned}
\{F, K\}=\int \mathrm{d}^{3} z & -R^{i(f)}\left(f \partial_{i} k-k \partial_{i} f\right)-R_{i}^{(g)}\left(f^{j} \partial_{j} k^{i}-k^{j} \partial_{j} f^{i}\right) \\
+ & \left.2 m^{4} \sqrt{\operatorname{det} \gamma} U^{m n} \gamma_{n j}\left(k^{j} \partial_{m} f-f^{j} \partial_{m} k\right)\right] .
\end{aligned}
$$

Since $k^{i}=n^{i} k$ and $f^{i}=n^{i} f$, the second term in this expression is,

$$
-R_{i}^{(g)}\left(f^{j} \partial_{j} k^{i}-k^{j} \partial_{j} f^{i}\right)=n^{i} R_{i}^{(g)}\left(k^{j} \partial_{j} f-f^{j} \partial_{j} k\right) .
$$


By the definition of $U^{m n}$ (3.3), the last term in (B.62) is,

$$
\begin{aligned}
& 2 m^{4} \sqrt{\operatorname{det} \gamma} U^{m n} \gamma_{n j}\left(k^{j} \partial_{m} f-f^{j} \partial_{m} k\right) \\
& \quad=2 m^{4} \sqrt{\operatorname{det} \gamma} U\left(k^{j} \partial_{j} f-f^{j} \partial_{j} k\right)+4 m^{4} \sqrt{\operatorname{det} \gamma} \frac{\partial U}{\partial \gamma_{m n}} \gamma_{n j}\left(k^{j} \partial_{m} f-f^{j} \partial_{m} k\right) .
\end{aligned}
$$

Then, using the expression for $U$ in (3.28) we have,

$$
\begin{aligned}
2 m^{4} \sqrt{\operatorname{det} \gamma} U\left(k^{j} \partial_{j} f-f^{j} \partial_{j} k\right)= & 2 m^{4} \sqrt{\operatorname{det} \gamma}\left[\frac{\beta_{1}}{\sqrt{x}}+\beta_{2} D_{l}^{l}+\beta_{3} \sqrt{x} e_{2}(D)\right]\left(k^{j} \partial_{j} f-f^{j} \partial_{j} k\right) \\
& -n^{i} R_{i}^{(g)}\left(k^{j} \partial_{j} f-f^{j} \partial_{j} k\right) .
\end{aligned}
$$

The last term cancels against the expression in (B.63), so that (B.62) becomes,

$$
\begin{aligned}
\{F, K\}= & \int \mathrm{d}^{3} z\left[-\left(f R^{i(f)} \partial_{i} k-k R^{i(f)} \partial_{i} f\right)\right. \\
& +2 m^{4} \sqrt{\operatorname{det} \gamma}\left(\frac{\beta_{1}}{\sqrt{x}}+\beta_{2} D_{l}^{l}+\frac{\beta_{3}}{2} \sqrt{x}\left(D^{k}{ }_{k} D_{r}^{r}-D_{r}^{k} D^{r}{ }_{k}\right)\right)\left(k^{j} \partial_{j} f-f^{j} \partial_{j} k\right) \\
& \left.+4 m^{4} \sqrt{\operatorname{det} \gamma} \frac{\partial U}{\partial \gamma_{m n}} \gamma_{n j}\left(k^{j} \partial_{m} f-f^{j} \partial_{m} k\right)\right]
\end{aligned}
$$

We now concentrate on the last term of (B.66). The $\beta_{2}$-part of this, using equation (3.30), becomes,

$$
\left[4 m^{4} \sqrt{\operatorname{det} \gamma} \frac{\partial U}{\partial \gamma_{m n}} \gamma_{n j}\left(k^{j} \partial_{m} f-f^{j} \partial_{m} k\right)\right]_{\beta_{2}}=-2 m^{4} \sqrt{\operatorname{det} \gamma} \beta_{2} D_{j}^{m}\left(k^{j} \partial_{m} f-f^{j} \partial_{m} k\right),
$$

where we have used (2.17), together with the relation

$$
\phi_{n k}\left(D^{-1}\right)^{k} \gamma^{m l}=x D^{m}{ }_{n}+D^{m}{ }_{i} n^{i} n^{k} \phi_{k n},
$$

which follows from (2.16). The $\beta_{3}$-part takes the form, on using (3.31),

$$
\begin{gathered}
{\left[4 m^{4} \sqrt{\operatorname{det} \gamma} \frac{\partial U}{\partial \gamma_{m n}} \gamma_{n j}\left(k^{j} \partial_{m} f-f^{j} \partial_{m} k\right)\right]_{\beta_{3}}=2 m^{4} \sqrt{\operatorname{det} \gamma} \beta_{3}\left[\sqrt{x} D_{l}^{l} \phi_{i r}\left(D^{-1}\right)_{k}^{r}-\sqrt{x} \phi_{i k}\right.} \\
\left.+\frac{1}{\sqrt{x}} n^{l} \phi_{l q} D^{q}{ }_{r} n^{r} \phi_{i s}\left(D^{-1}\right)^{s}{ }_{k}-\frac{1}{\sqrt{x}} n^{r} \phi_{r i} n^{l} \phi_{l k}\right] \frac{\partial \gamma^{i k}}{\partial \gamma_{m n}} \gamma_{n j}\left(k^{j} \partial_{m} f-f^{j} \partial_{m} k\right) .
\end{gathered}
$$

Using (2.17), (B.48) and (B.68), many of these terms cancel in a way similar to the $\beta_{2}$ case in (B.67). After some calculations, the $\beta_{3}$-part reduces to

$$
\begin{aligned}
& {\left[4 m^{4} \sqrt{\operatorname{det} \gamma} \frac{\partial U}{\partial \gamma_{m n}} \gamma_{n j}\left(k^{j} \partial_{m} f-f^{j} \partial_{m} k\right)\right]_{\beta_{3}}} \\
& \quad=2 m^{4} \sqrt{\operatorname{det} \gamma} \beta_{3} \sqrt{x}\left(D^{m}{ }_{k} D^{k}{ }_{j}-D^{m}{ }_{j} D_{k}^{k}\right)\left(k^{j} \partial_{m} f-f^{j} \partial_{m} k\right) .
\end{aligned}
$$


Finally, combining these results with equation (2.30), and performing some manipulations, allows the bracket (B.66) to be expressed as,

$$
\begin{aligned}
\{F, K\} & =\int \mathrm{d}^{3} z\left[-\left(f R^{i(f)} \partial_{i} k-k R^{i(f)} \partial_{i} f\right)-\phi^{i j} R_{j}^{(g)}\left(f \partial_{i} k-k \partial_{i} f\right)\right] \\
& =-\int \mathrm{d}^{3} z\left(f \tilde{R}^{i} \partial_{i} k-k \tilde{R}^{i} \partial_{i} f\right),
\end{aligned}
$$

where we have defined

$$
\tilde{R}^{i} \equiv \phi^{i j} \tilde{R}_{j}=\phi^{i j} R_{j}^{(g)}+R^{i(f)} .
$$

Combining this with (B.57) yields the bracket (4.5),

$$
\left\{\tilde{R}^{0}(x), \tilde{R}^{0}(y)\right\}=-\left(\tilde{R}^{i}(x) \frac{\partial}{\partial x^{i}} \delta^{3}(x-y)-\tilde{R}^{i}(y) \frac{\partial}{\partial y^{i}} \delta^{3}(x-y)\right) .
$$

\section{B.3 Poisson brackets with the constraint $\mathcal{C}$}

We will now compute the Poisson brackets of $\tilde{R}^{0}$ and $\tilde{R}_{i}$ with $\mathcal{C}$ in $(2.22)$. Let us define,

$$
F_{C} \equiv \int \mathrm{d}^{3} x f(x) \mathcal{C}(x) .
$$

The variations of $F_{C}$ with respect to $\gamma_{i j}, \pi^{i j}, \phi_{i j}$ and $p^{i j}$ are,

$$
\begin{array}{rlrl}
\frac{\delta F_{C}}{\delta \gamma_{m n}} & =\frac{\delta A^{0}}{\delta \gamma_{m n}}+\frac{\delta A}{\delta \gamma_{m n}}+2 m^{4} f \frac{\partial(\sqrt{\operatorname{det} \gamma} V)}{\partial \gamma_{m n}}, & & \frac{\delta F_{C}}{\delta \pi^{m n}}=\frac{\delta A^{0}}{\delta \pi^{m n}}+\frac{\delta A}{\delta \pi^{m n}}, \\
\frac{\delta F_{C}}{\delta \phi_{m n}}=\frac{\delta A}{\delta \phi_{m n}}+2 m^{4} f \sqrt{\operatorname{det} \gamma} \frac{\partial V}{\partial \phi_{m n}}, & \frac{\delta F_{C}}{\delta p^{m n}}=0
\end{array}
$$

where,

$$
A^{0} \equiv \int \mathrm{d}^{3} x f(x) R^{0(g)}(x), \quad A \equiv \int \mathrm{d}^{3} x f(x) R_{i}^{(g)}(x) D^{i}{ }_{j}(x) n^{j}(x) .
$$

Now, for $G$ defined in (B.12), we evaluate the bracket $\left\{F_{C}, G\right\}$ by spliting it in two parts,

$$
\left\{F_{C}, G\right\}=\left\{F_{C}, G\right\}_{g}+\left\{F_{C}, G\right\}_{f} .
$$

The $g$-bracket is given by,

$$
\left\{F_{C}, G\right\}_{g}=\left\{A^{0}, G\right\}_{g}+\{A, G\}_{g}+2 m^{4} \int \mathrm{d}^{3} z f \frac{\partial(\sqrt{\operatorname{det} \gamma} V)}{\partial \gamma_{m n}} \frac{\delta G}{\delta \pi^{m n}} .
$$

The first bracket in this expression is similar to (B.8), and therefore becomes

$$
\left\{A^{0}, G\right\}_{g}=-\int \mathrm{d}^{3} z f \nabla_{j}\left(g^{j} R^{0(g)}\right)=-\int \mathrm{d}^{3} z f \partial_{i}\left(g^{i} R^{0(g)}\right) .
$$

The last step holds since $g^{i}$ is a vector and $R^{0(g)}$ is a scalar density, in analogy with (B.28). The second bracket is similar to (B.7), but there will be an extra term due to the fact that the variation of $D^{i}{ }_{j} n^{j}$ with respect to $\gamma_{i j}$ does not vanish. The bracket therefore becomes

$$
\{A, G\}_{g}=-\int \mathrm{d}^{3} z\left(f D^{i}{ }_{j} n^{j} R_{k}^{(g)} \nabla_{i} g^{k}-g^{k} R_{i}^{(g)} \nabla_{k}\left(f D^{i}{ }_{j} n^{j}\right)-f R_{i}^{(g)} \frac{\partial\left(D^{i}{ }_{j} n^{j}\right)}{\partial \gamma_{m n}} \frac{\delta G}{\delta \pi^{m n}}\right) .
$$


The $f$-bracket $\left\{F_{C}, G\right\}_{f}$ is given by,

$$
\left\{F_{C}, G\right\}_{f}=\int \mathrm{d}^{3} z\left[f R_{i}^{(g)} \frac{\partial\left(D^{i}{ }_{j} n^{j}\right)}{\partial \phi_{m n}} \frac{\delta G}{\delta p^{m n}}+2 m^{4} f \sqrt{\operatorname{det} \gamma} \frac{\partial V}{\partial \phi_{m n}} \frac{\delta G}{\delta p^{m n}}\right] .
$$

Putting these together gives,

$$
\begin{array}{r}
\left\{F_{C}, G\right\}=\int \mathrm{d}^{3} z\left[g^{k} R_{i}^{(g)} \nabla_{k}\left(f D^{i}{ }_{j} n^{j}\right)-f \partial_{j}\left(g^{j} R^{0(g)}\right)-f D^{i}{ }_{j} n^{j} R_{k}^{(g)} \nabla_{i} g^{k}\right. \\
\left.+f S^{m n} \frac{\delta G}{\delta \pi^{m n}}+f Z^{m n} \frac{\delta G}{\delta p^{m n}}\right]
\end{array}
$$

Here, $S^{m n}$ is defined in $(2.37)$ and $Z^{m n}$ is defined in (3.5). The first term can be rewritten as,

$$
\int \mathrm{d}^{3} z g^{k} R_{i}^{(g)} \nabla_{k}\left(f D^{i}{ }_{j} n^{j}\right)=-\int \mathrm{d}^{3} z f \partial_{i}\left(g^{i} R_{j}^{(g)} D^{j}{ }_{k} n^{k}\right)+\int \mathrm{d}^{3} z f g^{k} R_{i}^{(g)} \nabla_{k}\left(D^{i}{ }_{j} n^{j}\right) .
$$

The expression for the variation of $G$ given in (B.16), together with (2.39), allows us to write

$$
f S^{m n} \frac{\delta G}{\delta \pi^{m n}}=-2 m^{4} f \sqrt{\operatorname{det} \gamma} V \nabla_{j} g^{j}+2 m^{4} f \sqrt{\operatorname{det} \gamma} \bar{V}^{m n} \gamma_{n j} \nabla_{m} g^{j},
$$

where the first term can be further simplified using,

$$
\sqrt{\operatorname{det} \gamma} V \nabla_{j} g^{j}=\partial_{i}\left(g^{i} \sqrt{\operatorname{det} \gamma} V\right)-g^{i} \sqrt{\operatorname{det} \gamma} \partial_{i} V .
$$

In a similar way, using (3.14) and (B.18), we can write

$$
f Z^{m n} \frac{\delta G}{\delta p^{m n}}=-2 m^{4} f \sqrt{\operatorname{det} \gamma} \bar{W}^{m n} \phi_{n j} \bar{\nabla}_{m} g^{j} .
$$

Putting all of this together, the bracket $\left\{F_{C}, G\right\}$ becomes

$$
\left\{F_{C}, G\right\}=\int \mathrm{d}^{3} z f\left[-\partial_{i}\left(g^{i} \mathcal{C}\right)+\Delta^{\prime}\right]
$$

where $\Delta^{\prime}$ is the same as the quantity $\Delta$ in (3.25) with $L^{i}$ replaced by $g^{i}$,

$$
\begin{aligned}
\Delta^{\prime}= & R_{j}^{(g)}\left(\nabla_{i}\left(D^{j}{ }_{k} n^{k}\right) g^{i}-D^{i}{ }_{k} n^{k} \nabla_{i} g^{j}\right) \\
& +2 m^{4} \sqrt{\operatorname{det} \gamma}\left(\partial_{i} V g^{i}+\gamma_{j k} \bar{V}^{k i} \nabla_{i} g^{j}-\phi_{j k} \bar{W}^{k i} \bar{\nabla}_{i} g^{j}\right) .
\end{aligned}
$$

We now show that $\Delta^{\prime}=0$. First, note that the Christoffel symbols in the first two terms cancel,

$$
R_{j}^{(g)}\left(\nabla_{i}\left(D^{j}{ }_{k} n^{k}\right) g^{i}-D^{i}{ }_{k} n^{k} \nabla_{i} g^{j}\right)=R_{j}^{(g)}\left(\partial_{i}\left(D^{j}{ }_{k} n^{k}\right) g^{i}-D^{i}{ }_{k} n^{k} \partial_{i} g^{j}\right) .
$$

The last two terms of $\Delta^{\prime}$ are,

$$
\gamma_{j k} \bar{V}^{k i} \nabla_{i} g^{j}-\phi_{j k} \bar{W}^{k i} \bar{\nabla}_{i} g^{j}=\left(\bar{V}^{k i} \gamma_{j k}-\bar{W}^{k i} \phi_{j k}\right) \partial_{i} g^{j}+\left(\bar{V}^{k i} \gamma_{j k} \Gamma_{i l}^{j}-\bar{W}^{k i} \phi_{j k} \bar{\Gamma}_{i l}^{j}\right) g^{l} .
$$


Using the symmetry relation (2.18), it follows from (2.40) and (3.13) that

$$
\begin{aligned}
\bar{V}^{k i} \gamma_{j k}-\bar{W}^{k i} \phi_{j k}= & \frac{n^{k} \phi_{k m}}{\sqrt{x}} D^{i}{ }_{l} n^{l}\left[\beta_{1} \delta^{m}{ }_{j}+\beta_{2} \sqrt{x}\left(\delta^{m}{ }_{j} D_{r}^{r}-D^{m}{ }_{j}\right)\right. \\
& \left.+\beta_{3} x\left(\frac{1}{2} \delta^{m}{ }_{j}\left(D^{r}{ }_{r} D^{h}{ }_{h}-D^{r}{ }_{h} D^{h}{ }_{r}\right)+D^{m}{ }_{n} D^{n}{ }_{j}-D^{m}{ }_{j} D^{r}{ }_{r}\right)\right],
\end{aligned}
$$

which, together with (2.28) and (2.30), implies that

$$
2 m^{4} \sqrt{\operatorname{det} \gamma}\left(\bar{V}^{k i} \gamma_{j k}-\bar{W}^{k i} \phi_{j k}\right)=R_{j}^{(g)} D^{i}{ }_{k} n^{k} .
$$

Also, since $\bar{V}^{k i}$ and $\bar{W}^{k i}$ are symmetric, the last two terms in (B.91) become,

$$
\bar{V}^{k i} \gamma_{j k} \Gamma_{i l}^{j}=\frac{1}{2} \bar{V}^{k i} \partial_{l} \gamma_{i k}, \quad \bar{W}^{k i} \phi_{j k} \bar{\Gamma}_{i l}^{j}=\frac{1}{2} \bar{W}^{k i} \partial_{l} \phi_{i k} .
$$

The above equations can be used to rewrite $\Delta^{\prime}$ as,

$$
\Delta^{\prime}=R_{j}^{(g)} \partial_{i}\left(D^{j}{ }_{k} n^{k}\right) g^{i}+m^{4} \sqrt{\operatorname{det} \gamma}\left[2 \partial_{i} V g^{i}+\left(\bar{V}^{m n} \partial_{i} \gamma_{m n}-\bar{W}^{m n} \partial_{i} \phi_{m n}\right) g^{i}\right] .
$$

We will now compute $\partial_{i} V$. From (2.25) it follows that,

$$
\begin{aligned}
\partial_{i} V= & \beta_{1} \partial_{i}\left(\sqrt{x} D^{j}{ }_{j}\right)+\frac{1}{2} \beta_{2}\left(2 \sqrt{x} D^{j}{ }_{j} \partial_{i}\left(\sqrt{x} D_{k}^{k}\right)-\partial_{i}\left(x D^{j}{ }_{k} D^{k}{ }_{j}\right)\right) \\
& +\frac{1}{6} \beta_{3}\left(3 x D_{j}^{j} D^{k}{ }_{k} \partial_{i}\left(\sqrt{x} D^{l}{ }_{l}\right)-3 x D^{k}{ }_{l} D^{l}{ }_{k} \partial_{i}\left(\sqrt{x} D^{j}{ }_{j}\right)\right. \\
& \left.-3 \sqrt{x} D^{j}{ }_{j} \partial_{i}\left(x D^{k}{ }_{l} D_{k}^{l}\right)+2 \partial_{i}\left(x^{3 / 2} D^{j}{ }_{k} D^{k}{ }_{l} D^{l}{ }_{j}\right)\right) .
\end{aligned}
$$

To evaluate this, we need the following derivatives that can be computed using equation (2.16),

$$
\begin{aligned}
\partial_{i}\left(\sqrt{x} D^{j}{ }_{j}\right) & =\frac{1}{2} \frac{1}{\sqrt{x}}\left[-2 n^{j} \phi_{l j} \partial_{i}\left(D_{n}^{l} n^{n}\right)-\phi_{l j}\left(D^{-1}\right)^{j}{ }_{k} \gamma^{k m} \gamma^{l n} \partial_{i} \gamma_{m n}+Q_{1}^{j l} \partial_{i} \phi_{l j}\right], \\
\partial_{i}\left(x D^{j}{ }_{k} D^{k}{ }_{j}\right) & =-2 D^{j}{ }_{m} n^{m} \phi_{l j} \partial_{i}\left(D^{l}{ }_{n} n^{n}\right)-\phi_{l j} \gamma^{j m} \gamma^{l n} \partial_{i} \gamma_{m n}+Q_{2}^{j l} \partial_{i} \phi_{l j}, \\
\partial_{i}\left(x^{3 / 2} D^{j}{ }_{k} D^{k}{ }_{l} D^{l}{ }_{j}\right) & =\frac{3}{2} \sqrt{x}\left[-2 D^{j}{ }_{k} D^{k}{ }_{m} n^{m} \phi_{l j} \partial_{i}\left(D^{l}{ }_{n} n^{n}\right)-\phi_{l j} D^{j}{ }_{k} \gamma^{k m} \gamma^{l n} \partial_{i} \gamma_{m n}+Q_{3}^{j l} \partial_{i} \phi_{l j}\right] .
\end{aligned}
$$

Using these expressions, as well as (2.40) and (3.13), equation (B.96) can be rewritten as

$$
\begin{aligned}
\partial_{i} V= & -\left[\frac{\beta_{1}}{\sqrt{x}} n^{j} \phi_{l j}+\beta_{2}\left(D^{r}{ }_{r} n^{j} \phi_{l j}-D^{j}{ }_{m} n^{m} \phi_{l j}\right)\right. \\
& \left.+\beta_{3} \sqrt{x}\left(\frac{1}{2}\left(D^{r}{ }_{r} D^{h}{ }_{h}-D^{r}{ }_{h} D^{h}{ }_{r}\right) n^{j} \phi_{l j}-D^{r}{ }_{r} D^{j}{ }_{m} n^{m} \phi_{l j}+D^{j}{ }_{k} D^{k}{ }_{m} n^{m} \phi_{l j}\right)\right] \partial_{i}\left(D^{l}{ }_{n} n^{n}\right) \\
& -\frac{1}{2} \bar{V}^{m n} \partial_{i} \gamma_{m n}+\frac{1}{2} \bar{W}^{m n} \partial_{i} \phi_{m n},
\end{aligned}
$$

which, together with (2.18) and (2.28) implies

$$
2 m^{4} \sqrt{\operatorname{det} \gamma} \partial_{i} V=-R_{j}^{(g)} \partial_{i}\left(D^{j}{ }_{k} n^{k}\right)-m^{4} \sqrt{\operatorname{det} \gamma}\left(\bar{V}^{m n} \partial_{i} \gamma_{m n}-\bar{W}^{m n} \partial_{i} \phi_{m n}\right) .
$$


Substituting this in (B.95) gives $\Delta^{\prime}=0$, as desired, and one obtains,

$$
\left\{F_{C}, G\right\}=-\int \mathrm{d}^{3} z f \partial_{i}\left(g^{i} \mathcal{C}\right)
$$

This expression is of the same form as (B.54), but with $\tilde{R}^{0}$ replaced by $\mathcal{C}$. By the same argument as for that bracket, it follows that

$$
\left\{\mathcal{C}(x), \tilde{R}_{i}(y)\right\}=-\mathcal{C}(y) \frac{\partial}{\partial x^{i}} \delta^{3}(x-y) .
$$

Since $\mathcal{C}$ is a scalar density, it should transform as such under spatial diffeomorphisms. Note that the Poisson bracket above is of the same form as (4.4). Since $\tilde{R}^{0}$ is also a scalar density, this means that $\mathcal{C}$ transforms in the appropriate way, consistent with our earlier results.

Finally, we look at the bracket $\left\{\mathcal{C}(x), \tilde{R}^{0}(y)\right\}$. Recall from (3.1) that

$$
\mathcal{C}_{(2)}(x) \approx-\int \mathrm{d}^{3} y\left[L^{i}(y)\left\{\mathcal{C}(x), \tilde{R}_{i}(y)\right\}+L(y)\left\{\mathcal{C}(x), \tilde{R}^{0}(y)\right\}\right] .
$$

Imposing the constraint $\mathcal{C}=0$, equation (B.101) implies that

$$
\int \mathrm{d}^{3} y L(y)\left\{\mathcal{C}(x), \tilde{R}^{0}(y)\right\}=-\mathcal{C}_{(2)}(x)+L^{i}(x) \frac{\partial}{\partial x^{i}} \mathcal{C}(x) \approx-\mathcal{C}_{(2)}(x),
$$

since the spatial derivative of $\mathcal{C}$ vanishes on the constraint surface. In particular, equation (B.103) shows that $\mathcal{C}_{(2)}$ is independent of $L^{i}$, as discussed in section 3.2. Then it follows that computing the bracket $\left\{\mathcal{C}(x), \tilde{R}^{0}(y)\right\}$ involves preforming essentially the same calculations as those in section 3 . The result is

$$
\left\{\mathcal{C}(x), \tilde{R}^{0}(y)\right\}=-\frac{\mathcal{C}_{(2)}(x)}{L(x)} \delta^{3}(x-y) .
$$

The right hand side of (B.104) is independent of $L$, as can be seen from (3.35).

Open Access. This article is distributed under the terms of the Creative Commons Attribution License (CC-BY 4.0), which permits any use, distribution and reproduction in any medium, provided the original author(s) and source are credited.

\section{References}

[1] D.G. Boulware and S. Deser, Can gravitation have a finite range?, Phys. Rev. D 6 (1972) 3368 [InSPIRE].

[2] S.F. Hassan and R.A. Rosen, Bimetric Gravity from Ghost-free Massive Gravity, JHEP 02 (2012) 126 [arXiv:1109.3515] [INSPIRE].

[3] S.F. Hassan and M. Kocic, On the local structure of spacetime in ghost-free bimetric theory and massive gravity, JHEP 05 (2018) 099 [arXiv: 1706.07806] [INSPIRE].

[4] S.F. Hassan and R.A. Rosen, Confirmation of the Secondary Constraint and Absence of Ghost in Massive Gravity and Bimetric Gravity, JHEP 04 (2012) 123 [arXiv:1111.2070] [INSPIRE].

[5] S.A. Hojman, K. Kuchar and C. Teitelboim, Geometrodynamics Regained, Annals Phys. 96 (1976) 88 [inSPIRE]. 
[6] C. de Rham and G. Gabadadze, Generalization of the Fierz-Pauli Action, Phys. Rev. D 82 (2010) 044020 [arXiv: 1007.0443] [InSPIRE].

[7] C. de Rham, G. Gabadadze and A.J. Tolley, Resummation of Massive Gravity, Phys. Rev. Lett. 106 (2011) 231101 [arXiv:1011.1232] [INSPIRE].

[8] S.F. Hassan and R.A. Rosen, On Non-Linear Actions for Massive Gravity, JHEP 07 (2011) 009 [arXiv: 1103.6055] [INSPIRE].

[9] S.F. Hassan and R.A. Rosen, Resolving the Ghost Problem in non-Linear Massive Gravity, Phys. Rev. Lett. 108 (2012) 041101 [arXiv:1106.3344] [INSPIRE].

[10] S.F. Hassan, R.A. Rosen and A. Schmidt-May, Ghost-free Massive Gravity with a General Reference Metric, JHEP 02 (2012) 026 [arXiv:1109.3230] [INSPIRE].

[11] D. Comelli, M. Crisostomi, F. Nesti and L. Pilo, Degrees of Freedom in Massive Gravity, Phys. Rev. D 86 (2012) 101502 [arXiv:1204.1027] [INSPIRE].

[12] D. Comelli, F. Nesti and L. Pilo, Massive gravity: a General Analysis, JHEP 07 (2013) 161 [arXiv: 1305.0236] [INSPIRE].

[13] S.F. Hassan, A. Schmidt-May and M. von Strauss, Proof of Consistency of Nonlinear Massive Gravity in the Stúckelberg Formulation, Phys. Lett. B 715 (2012) 335 [arXiv: 1203.5283] [INSPIRE].

[14] C. Deffayet, J. Mourad and G. Zahariade, Covariant constraints in ghost free massive gravity, JCAP 01 (2013) 032 [arXiv: 1207.6338] [INSPIRE].

[15] L. Bernard, C. Deffayet and M. von Strauss, Consistent massive graviton on arbitrary backgrounds, Phys. Rev. D 91 (2015) 104013 [arXiv:1410.8302] [INSPIRE].

[16] L. Bernard, C. Deffayet and M. von Strauss, Massive graviton on arbitrary background: derivation, syzygies, applications, JCAP 06 (2015) 038 [arXiv: 1504.04382] [INSPIRE].

[17] S. Deser, M. Sandora, A. Waldron and G. Zahariade, Covariant constraints for generic massive gravity and analysis of its characteristics, Phys. Rev. D 90 (2014) 104043 [arXiv: 1408.0561] [INSPIRE].

[18] T. Kugo and N. Ohta, Covariant Approach to the No-ghost Theorem in Massive Gravity, PTEP 2014 (2014) 043B04 [arXiv:1401.3873] [INSPIRE].

[19] J. Kluson, Is Bimetric Gravity Really Ghost Free?, Int. J. Mod. Phys. A 28 (2013) 1350143 [arXiv:1301.3296] [INSPIRE].

[20] J. Kluson, Hamiltonian Formalism of General Bimetric Gravity, Eur. Phys. J. C 73 (2013) 2553 [arXiv:1303.1652] [inSPIRE].

[21] J. Klusoň, Hamiltonian Formalism of Bimetric Gravity In Vierbein Formulation, Eur. Phys. J. C 74 (2014) 2985 [arXiv: 1307.1974] [InSPIRE].

[22] S. Alexandrov, K. Krasnov and S. Speziale, Chiral description of ghost-free massive gravity, JHEP 06 (2013) 068 [arXiv:1212.3614] [INSPIRE].

[23] S. Alexandrov, Canonical structure of Tetrad Bimetric Gravity, Gen. Rel. Grav. 46 (2014) 1639 [arXiv:1308.6586] [INSPIRE].

[24] V.O. Soloviev and M.V. Tchichikina, Bigravity in Kuchar's Hamiltonian formalism: The General Case, Theor. Math. Phys. 176 (2013) 1163 [arXiv:1211.6530] [InSPIRE].

[25] V.O. Soloviev and M.V. Tchichikina, Bigravity in Kuchar's Hamiltonian formalism. 2. The special case, Phys. Rev. D 88 (2013) 084026 [arXiv:1302.5096] [INSPIRE]. 
[26] L. Bernard, C. Deffayet, A. Schmidt-May and M. von Strauss, Linear spin-2 fields in most general backgrounds, Phys. Rev. D 93 (2016) 084020 [arXiv:1512.03620] [INSPIRE].

[27] P. Creminelli, A. Nicolis, M. Papucci and E. Trincherini, Ghosts in massive gravity, JHEP 09 (2005) 003 [hep-th/0505147] [INSPIRE].

[28] M.S. Volkov, Stability of Minkowski space in ghost-free massive gravity theory, Phys. Rev. D 90 (2014) 024028 [arXiv: 1402.2953] [INSPIRE].

[29] M.S. Volkov, Energy in ghost-free massive gravity theory, Phys. Rev. D 90 (2014) 124090 [arXiv: 1404.2291] [INSPIRE].

[30] C. Teitelboim, How commutators of constraints reflect the space-time structure, Annals Phys. 79 (1973) 542 [inSPIRE].

[31] S.F. Hassan, A. Schmidt-May and M. von Strauss, On Consistent Theories of Massive Spin-2 Fields Coupled to Gravity, JHEP 05 (2013) 086 [arXiv:1208.1515] [INSPIRE].

[32] K. Izumi and Y.C. Ong, An analysis of characteristics in nonlinear massive gravity, Class. Quant. Grav. 30 (2013) 184008 [arXiv: 1304.0211] [INSPIRE].

[33] X.O. Camanho, G. Lucena Gómez and R. Rahman, Causality Constraints on Massive Gravity, Phys. Rev. D 96 (2017) 084007 [arXiv: 1610.02033] [INSPIRE].

[34] B. Bellazzini, F. Riva, J. Serra and F. Sgarlata, Beyond Positivity Bounds and the Fate of Massive Gravity, Phys. Rev. Lett. 120 (2018) 161101 [arXiv:1710.02539] [InSPIRE].

[35] K. Hinterbichler, A. Joyce and R.A. Rosen, Massive Spin-2 Scattering and Asymptotic Superluminality, JHEP 03 (2018) 051 [arXiv: 1708.05716] [INSPIRE].

[36] J. Bonifacio, K. Hinterbichler, A. Joyce and R.A. Rosen, Massive and Massless Spin-2 Scattering and Asymptotic Superluminality, JHEP 06 (2018) 075 [arXiv:1712.10020] [INSPIRE].

[37] K. Hinterbichler, A. Joyce and R.A. Rosen, Eikonal scattering and asymptotic superluminality of massless higher spin fields, Phys. Rev. D 97 (2018) 125019 [arXiv: 1712.10021] [INSPIRE].

[38] R.L. Arnowitt, S. Deser and C.W. Misner, The Dynamics of general relativity, Gen. Rel. Grav. 40 (2008) 1997 [gr-qc/0405109] [INSPIRE].

[39] S.F. Hassan, M. Kocic and A. Schmidt-May, Absence of ghost in a new bimetric-matter coupling, arXiv:1409.1909 [INSPIRE].

[40] P.A.M. Dirac, Lectures on quantum mechanics, Belfer Graduate School of Science Monographs Series, vol. 2, Belfer Graduate School of Science, New York (1964).

[41] B.S. DeWitt, Quantum Theory of Gravity. 1. The Canonical Theory, Phys. Rev. 160 (1967) 1113 [INSPIRE].

[42] P. Peldan, Ashtekar's variables for arbitrary gauge group, Phys. Rev. D 46 (1992) R2279 [hep-th/9204069] [INSPIRE].

[43] R.L. Arnowitt, S. Deser and C.W. Misner, Coordinate invariance and energy expressions in general relativity, Phys. Rev. 122 (1961) 997 [INSPIRE].

[44] T. Regge and C. Teitelboim, Role of Surface Integrals in the Hamiltonian Formulation of General Relativity, Annals Phys. 88 (1974) 286 [InSPIRE].

[45] J. Khoury, G.E.J. Miller and A.J. Tolley, Spatially Covariant Theories of a Transverse, Traceless Graviton, Part I: Formalism, Phys. Rev. D 85 (2012) 084002 [arXiv:1108.1397] [INSPIRE]. 